Revista Mexicana de Economía y Finanzas Nueva Época Volumen 14 Número 3, Julio-Septiembre 2019, pp. 353-377

DOI: https://doi.org/10.21919/remef.v14i3.308

\title{
Costo de generación eléctrica incorporando externalidades ambientales: Mezcla óptima de tecnologías de carga base
}

\author{
María del Carmen Gómez-Ríos \\ Universidad Anáhuac México-Norte, México \\ David Juárez-Luna ${ }^{1}$ \\ Universidad Anáhuac México-Norte, México \\ (Recepción: 18/junio/2018, aceptado: 7/diciembre/2018)
}

\section{Resumen}

El objetivo de este artículo es calcular el Costo Total Nivelado de Generación con Externalidades (CTNGE) de tres tecnologías de carga base: termoeléctrica de carbón, ciclo combinado y central nuclear. Se emplea simulación Monte Carlo para estimar las densidades de probabilidad de los CTNGE. Se emplea la teoría de portafolio para encontrar la mezcla de tecnologías que brinden el CTNGE menos riesgoso y con menor media. Se encuentra que la central nuclear tiene los menores CTNGE. Siendo la termoeléctrica de carbón la tecnología con mayores y más riesgosos CTNGE. El análisis sugiere que, al generar electricidad, conviene dejar fuera a la termoeléctrica de carbón y centrarse en dos tecnologías: ciclo combinado y central nuclear, asignando a ésta última una mayor participación. Una limitante del trabajo es que las densidades de probabilidad de los CTNGE estimadas a través de la simulación Monte Carlo dependen de los datos empleados. El presente análisis sugiere que el CTNGE se puede modificar significativamente al incluir el costo del $\mathrm{CO}_{2}$.

Clasificación JEL: D81, G11, Q40, Q53

Palabras clave: Emisiones de $\mathrm{CO}_{2}$, Generación, Electricidad, Costos nivelados

\section{Cost of electric generation accounting for environmental externalities: Optimal mix of baseload technologies}

\section{Abstract}

This paper aims to calculate the Total Levelized Cost of Generation with Externalities (CTNGE, in Spanish) of three baseload technologies: coal thermoelectric, combined cycle and nuclear power plant. Monte Carlo simulation is used to estimate the CTNGE probability densities. The portfolio theory is used to find the mix of technologies that provides the least risky CTNGE and with the lowest average. We find that the nuclear power plant has the lowest CTNGE. The coal-fired thermoelectric plant is the technology with the largest and riskiest CTNGE. The analysis suggests that, when generating electricity, it is convenient to leave out the coal-fired thermoelectric plant and focus on two technologies: combined cycle and nuclear power plant, assigning a higher participation to the latter. One limitation of the work is that the probability densities of the CTNGE estimated through the Monte Carlo simulation depend on the data used. The present analysis suggests that the CTNGE can be significantly modified by including the cost of $\mathrm{CO}_{2}$.

JEL Classification: D81, G11, Q40, Q53

Keywords: $\mathrm{CO}_{2}$ Emissions, Generation, Electricity, Levelized Cost

\footnotetext{
${ }^{1}$ Quinto piso del CAIDE. Universidad Anáhuac México, campus Norte. Av. Universidad Anáhuac 46, Col. Lomas Anáhuac, Huixquilucan, Estado de México, México. C. P 52786. Teléfono: 56270210. Ext. 8344. E-mail: david.juarez@anahuac.mx

* Sin fuente de financiamiento declarada para el desarrollo de la investigación
} 
REMEF (The Mexican Journal of Economics and Finance) Costo de generación eléctrica incorporando externalidades ambientales:

\section{Introducción}

La energía eléctrica se emplea para prácticamente todos los procesos de producción. Garantizar el suministro continuo, seguro y a costos adecuados de la misma incentiva el crecimiento económico (IEA, 2014²). Sin embargo, a pesar de su gran utilidad, la energía eléctrica, independientemente de la tecnología que se emplee para su generación, tiene un impacto ambiental a través de las externalidades originadas, principalmente, por las emisiones de $\mathrm{CO}_{2}$.

En el año 2015, el sector con mayores emisiones de $\mathrm{CO}_{2}$ correspondió a la generación de electricidad, representando el $42 \%$ de las emisiones totales. Donde las tecnologías que emplean combustibles fósiles, carbón y gas natural, contribuyeron a generar el $66 \%$ de la energía eléctrica mundial (IEA, 2017). Pese a ello, la mayor parte de los proyectos enfocados a generación de electricidad, solo han tomado en cuenta los costos privados, dejando de lado los daños ocasionados al medio ambiente. A este respecto, se ha mostrado que las tecnologías de generación de electricidad que emplean combustibles fósiles son las más contaminantes al mostrar los factores de emisión de $\mathrm{CO}_{2}$ más altos. El carbón produce más emisiones seguido por el gas natural (Spadaro et al., 2000). Lo que ha despertado un interés creciente, a nivel internacional, en los impactos ambientales causados por el sector eléctrico (IEA, 2001; NEA, 2003; ${ }^{3}$ IEA (2005). Mismo que se ve reflejado por el protocolo de Kioto, celebrado en el año 2008, y más recientemente, en el año 2015, se llevó a cabo el acuerdo de París. Ambos enfocados a mitigar las emisiones de $\mathrm{CO}_{2}$ (IEA, 2017).

Es de destacar que las diferentes tecnologías de generación de electricidad deberían incluir los costos externos relacionados con la salud humana y el medio ambiente (NEA, 2003). A pesar de que la energía nuclear no produce emisiones de $\mathrm{CO}_{2}$ en el proceso de generación de electricidad (Spadaro et al.,2000), es la única que incorpora, dentro del Costo Nivelado de Combustible, un monto destinado a cubrir los costos externos originados por el tratamiento y disposición del combustible gastado ${ }^{4}$ (IEA, 2015; Ramírez et al., 2006). Mientras que las centrales que utilizan combustibles fósiles, al no incorporar las externalidades, los costos de generación son menores. Lo que tiene como consecuencia que, los productores y consumidores de electricidad tengan la percepción errónea de que la energía eléctrica generada por una central nuclear es más costosa que la electricidad generada vía combustibles fósiles. Con lo que se tienen incentivos para no incorporar fuentes de energía bajas en emisiones de $\mathrm{CO}_{2}$, como es el caso de la energía nuclear.

Este hecho toma aún más relevancia ante los cambios que se han dado en la legislación de los sistemas eléctricos, a nivel nacional e internacional, que permiten la entrada a inversionistas privados a la generación de electricidad. Los inversionistas del sector privado, al contar con recursos limitados, desearán conocer en primera instancia los costos que enfrentan las centrales generadoras de energía eléctrica, incluyendo los costos externos.

Partiendo de lo anterior, el objetivo de este artículo es calcular el Costo Total Nivelado de Generación con Externalidades (CTNGE) de tres tecnologías de carga base: termoeléctrica de carbón, ciclo combinado y central nuclear. Se emplea simulación Monte Carlo para estimar las densidades de probabilidad de los CTNGE. De esta manera, se captura el carácter estocástico de sus componentes, a la vez que permite emplear la teoría de portafolio para encontrar la mezcla de tecnologías que brindan el CTNGE menos riesgoso y con menor media.

Los datos se toman de fuentes emitidas por la OCDE. Tales fueron IEA (2015) y OCDE (2017). También se tomaron datos del CENACE (2010-2012) ${ }^{5}$ CFE $(2014),{ }^{6}$ de

\footnotetext{
${ }^{2}$ Agencia Internacional de Energía (IEA, por sus siglas en inglés).

${ }^{3}$ Agencia de Energía Nuclear (NEA, por sus siglas en inglés).

${ }^{4}$ Ciclo cerrado del combustible nuclear (Ver Ramírez et al.,2006 para mayor detalle)

${ }^{5}$ Centro Nacional de Control de Energía.

${ }^{6}$ Comisión Federal de Electricidad.
} 
AEO $(2017)^{7}$ y del ICF Consulting Canada (2017).

Se obtienen tres conjuntos de resultados.

Primero, se emplea simulación Monte Carlo para generar variables aleatorias correspondientes a los CTNGE de las tres tecnologías. Se aplica entonces el concepto de dominancia estocástica de primer orden a las distribuciones acumuladas correspondientes. Se encontró que la central nuclear tiene, sin ambigüedades, los menores CTNGE. Siendo la termoeléctrica de carbón la tecnología con mayores CTNGE, que a la vez son los más riesgosos.

Segundo, se realiza un análisis de tipo tornado para identificar que tan sensibles son los CTNGE de las tres tecnologías, ante cambios en los parámetros de entrada. Se encontró que el costo de $\mathrm{CO}_{2}$ es el que más afecta a los CTNGE de la termoeléctrica de carbón y de ciclo combinado. Por su parte, el costo unitario de inversión es el que más afecta al CTNGE de la central nuclear.

En tercer lugar, se emplea la teoría de portafolio de Markowitz (1952) para encontrar la mezcla óptima de tecnologías de carga base. Se encontró que el portafolio que incluye a la central nuclear y al ciclo combinado es el que tiene el CTNGE con menor media y menor varianza. El análisis sugiere que, al generar electricidad, conviene dejar fuera a la termoeléctrica de carbón y centrarse en dos tecnologías: ciclo combinado y central nuclear, asignando a ésta última una proporción mayor de capital.

Estos resultados, particularmente el primero y el tercero, son importantes debido a que dan fundamentos para incrementar la generación eléctrica a través de centrales nucleares. A primera vista, esta sugerencia resulta contra intuitiva, dada la fuerte oposición social que enfrentan las centrales nucleares debido a, entre otras cosas, desastres como el ocurrido en Fukushima el 11 de marzo de $2011 .{ }^{8}$ No obstante, a diferencia de estudios previos, el presente análisis incluye los costos de las emisiones de $\mathrm{CO}_{2}$ al medio ambiente, lo que implica que los CTNGE del ciclo combinado y de la termoeléctrica de carbón son mayores al CTNGE de la central nuclear. Puede parecer que este resultado es esperado, debido a que la central nuclear no emite $\mathrm{CO}_{2}$ en su proceso de generación de electricidad. Sin embargo, la central nuclear también genera costos externos debido a los desechos del combustible. Mismos que se cubren destinando un monto del Costo Nivelado de Combustible al tratamiento y disposición del combustible gastado (Ramírez et al., 2006). Es decir, la central nuclear, aun cubriendo los costos externos ocasionados por los desechos del combustible, es la tecnología que tiene los CTNGE más bajos.

El presente artículo se enmarca en la literatura que analiza portafolios de inversión en generación de electricidad. Existen diversos estudios en esta línea de investigación. En su mayoría emplean Simulación Monte Carlo. No obstante, se enfocan principalmente en capturar el carácter estocástico de los precios de los combustibles y de la tarifa eléctrica. Para ello, se emplea principalmente el VPN, el costo de generación, rendimientos financieros, así como opciones reales (Ver, por ejemplo, Awerbuch y Berger, 2003; Roques, 2006; Roques et al., 2006; Vithayasrichareon et al., 2010a y Vithayasrichareon et al., 2010b). ${ }^{9}$

El presente trabajo se diferencia de los existentes en tres aspectos. Primero, toma como principales fuentes de incertidumbre al costo de las emisiones de $\mathrm{CO}_{2}$ y al costo unitario de inversión. Segundo, emplea el CTNGE para encontrar la mezcla óptima de tecnologías de carga base. Al igual que en los estudios previos, en el presente trabajo se emplea Simulación Monte Carlo. Tercero, el presente trabajo generaliza el "efecto portafolio" al encontrar el intervalo factible de capital asignado a cada tecnología que reduce el riesgo del CTNGE del portafolio.

\footnotetext{
${ }^{7}$ Perspectiva Anual de Energía (AEO, por sus siglas en inglés).

${ }^{8} \mathrm{La}$ electricidad generada por centrales nucleares también tiene características que la hacen poco atractiva (Ver Roques et al., 2006).

${ }^{9}$ Otro ejemplo de portafolios de inversión en generación eléctrica se encuentra en Kienzle y Andersson (2008).
} 
De los trabajos sobre portafolios de inversión en generación eléctrica destacan tres. Awerbuch y Berger (2003), con los objetivos de diversificar y de intensificar la seguridad energética, introducen el análisis de media-varianza de portafolios y lo aplican a la Unión Europea. Consideran que la incertidumbre proviene de tres fuentes: costo del combustible, costo de operación y mantenimiento y, costos de construcción. Se analizan portafolios de generación eléctrica que incluyen gas, carbón, energía nuclear, petróleo y un conjunto de energías renovables representadas por "viento". Para evaluar los proyectos de inversión en generación de electricidad se emplean rendimientos financieros. Encuentran que se puede reducir el riesgo y el costo del portafolio cuando se incluyen energías renovables que tengan costos fijos.

El segundo es el de Roques et al. (2006) quienes revisan algunas metodologías, tomadas de la literatura financiera, como la Simulación Monte Carlo, el análisis media-varianza de portafolios y las opciones reales como alternativas para evaluar proyectos de inversión en generación de electricidad. La viabilidad de las dos primeras metodologías se ilustra valuando portafolios que contienen tres tecnologías, las que emplean carbón, gas y energía nuclear. Para ello, emplean el VPN. Consideran que la incertidumbre proviene de tres factores: precios de los combustibles, tarifa eléctrica y precio del $\mathrm{CO}_{2}$. Muestran que introducir una central nuclear en un portafolio en el que domina el gas, mitiga la probabilidad de tener grandes pérdidas ocasionadas por la incertidumbre provocada por la volatilidad de los precios del gas y del carbón. Finalmente, discuten como las opciones reales pueden ayudar a capturar la flexibilidad gerencial y operacional de la inversión.

Más cercano al presente artículo se encuentra el trabajo de Vithayasrichareon et al. (2010b) quienes incluyen, entre otros factores, el precio del $\mathrm{CO}_{2}$ como fuente de incertidumbre. Ellos emplean simulación Monte Carlo para valuar diferentes portafolios que incluyen a la termoeléctrica de carbón, al ciclo combinado y la central nuclear. Para ello, emplean el costo de generación. Capturan el comportamiento estocástico del costo de los combustibles y de los costos de capital de las plantas en el contexto de la Asociación de Naciones del Sudeste Asiatico (ASEAN, por sus siglas en inglés). Encuentran que, a medida que el precio del $\mathrm{CO}_{2}$ aumenta, la viablilidad económica de la central nuclear aumenta en relación a la termoeléctrica de carbón y al ciclo combinado. Similar a los resultados obtenidos en el presente artículo, Vithayasrichareon et al. (2010b) sugieren que la energía nuclear tiene el potencial de incrementar la seguridad energética, a la vez que reduce los efectos que ocasiona la industria eléctrica al cambio climático. Esto se hará más evidente a medida que se empleen mecanismos adecuados para establecer precio a las emisiones de $\mathrm{CO}_{2}$.

La simulación Monte Carlo en evaluación de proyectos de generación eléctrica ha sido muy utilizada. Entre otras aplicaciones, se ha empleado para capturar los diferentes riesgos que implica el proyecto de una central nuclear (Rode et al., 2001), para evaluar un proyecto de inversión en genereación de eléctrica a través del viento (Khindanova, 2013), para simular la Producción Anual de Energía (PAE) eléctrica a través de la velocidad del viento (Hrafnkelsson et al., 2016).

El trabajo se divide en seis secciones. La primera presenta la metodología del CTNGE. La estimación del CTNGE empleando el método estocástico a las tres tecnologías de interés, se presenta en la segunda sección. En la tercera se estima el CTNGE a través de simulación Monte Carlo. En esta sección se emplea dominancia estocástica de primer orden para discriminar entre las distribuciones de los CTNGE de las tres tecnologías, a la par que se realiza análisis de sensibilidad de tipo tornado. La cuarta sección emplea teoría de portafolios para encontrar la mezcla óptima de tecnologías de carga base. En la quinta se presentan las conclusiones. La sección seis presenta recomendaciones y trabajos futuros. El apéndice contiene algunas demostraciones importantes. 


\section{Metodología del CTNGE}

El Costo Total Nivelado de Generación (CTNG) indica el costo por MWh generado. Es la metodología recomendada por los organismos nacionales e internacionales para calcular el costo de generación eléctrica (IEA, 2015). El CTNG se obtiene del cociente del valor presente de los egresos que ocasiona el diseño, la construcción de una central generadora y los costos durante su vida útil, entre el valor presente de la energía que aportará la central en dicho periodo (IEA, 2015 y CFE, 2014). Cabe destacar que, la metodología del CTNG descrita en IEA (2015) incluye el costo de las emisiones de $\mathrm{CO}_{2}$. De este modo, se tiene el CTNGE, que incorpora las afectaciones sociales debidas a externalidades negativas ocasionadas por la generación de electricidad.

En el presente trabajo se calcula el CTNGE de las tres tecnologías tradicionales de carga base, que son las que operan las 24 horas del día, los 365 días del año: a) termoeléctrica de carbón con capacidad de 1,131 MW; b) ciclo combinado que utiliza gas natural con 551 MW de capacidad, y; c) central nuclear equipada con un reactor $\mathrm{ABWR}^{10}$ de $1,425 \mathrm{MW}$ de capacidad instalada.

El CTNGE se compone de cuatro elementos: 1) Costo Nivelado de Inversión (CNI), se refiere a la cantidad monetaria destinada a la construcción de la central; 2) Costo Nivelado del Combustible (CNC), que abarca los costos de combustible; 3) El Costo Nivelado de las Externalidades por $\mathrm{CO}_{2}\left(\mathrm{CNCO}_{2}\right)$ busca compensar a la sociedad por las externalidades ocasionadas por las emisiones de $\mathrm{CO}_{2}$ en el proceso de generación de electricidad, y; 4) Costo Nivelado de Operación y Mantenimiento (CNOM) que cubre los costos de operación y mantenimiento por cada MWh generado (ver Gómez-Ríos, 2008 y Gómez-Ríos, 2016, para mayores detalles sobre el CTNGE). Así, el CTNGE se expresa como sigue:

$$
C T N G E=C N I+C N C+C N C O_{2}+C N O M
$$

En las siguientes secciones se calcularán, de manera detallada, los componentes del CTNGE para cada una de las tres tecnologías de carga base.

Es de hacer notar que el CTNGE tiene algunas limitaciones. El CTNGE brinda información que podría ser útil para que un inversionista debido al hecho de que a menores costos corresponden mayores ganancias. No obstante, esto no siempre se cumple ya que, en un mercado eléctrico liberalizado, la tarifa eléctrica es estocástica. De este modo, la ganancia de un inversionista, capturada por el Valor Presente Neto (VPN), dependerán del grado de correlación existente entre el costo de generación y la tarifa eléctrica (Roques et al.,2008). Adicionalmente, en un ambiente de incertidumbre, como el de generación eléctrica, existe un costo de oportunidad entre invertir hoy o esperar a invertir en una fecha posterior. Las Opciones Reales pueden ser empleadas para informar al inversionista sobre el momento exacto en el que debe invertir (Dixit y Pindyck, 1994). De este modo, la pérdida de información que implica el prescindir de la tarifa eléctrica y el costo de oportunidad entre invertir hoy o hacerlo en una fecha posterior, se compensa con la relativa simplicidad y manejabilidad del CTNGE, que ayuda a un inversionista a enfocarse en la tecnología de generación eléctrica de mínimo costo.

Como punto de partida, se comienza estimando el CTNGE empleando el método determinístico. Lo que brindará parámetros necesarios para calcular el CTNGE utilizando el método estocástico.

\section{Estimación del CTNGE utilizando el Método determinístico}

Para estimar el CTNGE de las tres tecnologías de interés, se comienza por describir las principales características de cada una de ellas.

A. Termoeléctrica de Carbón

Las termoeléctricas de carbón (TC) cuentan con las siguientes características: a) es

\footnotetext{
${ }^{10}$ Reactor avanzado de ebullición de agua (ABWR, por sus siglas en inglés).
} 
una tecnología intensiva en capital; b) la participación de los costos del combustible en su operación representa aproximadamente $40 \%$ de los costos totales; c) es una tecnología con una larga vida económica (40 años de operación), y; d) emite $\mathrm{CO}_{2}$ al ambiente en su proceso de generación de electricidad.

B. Ciclo Combinado

Las características del ciclo combinado (CC) son: a) es una tecnología que requiere un nivel relativamente bajo de inversión; b) la participación del costo del combustible es alta, alrededor de $65 \%$; c) tiene una vida económica de 30 años, y; d) emite dióxido de carbono $\left(\mathrm{CO}_{2}\right)$ al ambiente.

C. Central Nuclear

Para finalizar, las características de las centrales nucleares (CN) son: a) tecnología intensiva en capital (su participación dentro de los costos totales es de aproximadamente $60 \%$ ); b) la participación del costo de combustible respecto a los costos totales es relativamente bajo (17\%); c) es una tecnología con una larga vida económica (60 años de operación), y; d) no emite $\mathrm{CO}_{2}$ al ambiente.

A continuación, se estima el CTNGE de cada una de las tecnologías empleando el método determinístico. Para ello, se calculan los diferentes costos que lo componen: CNI, $\mathrm{CNC}$ ), $\mathrm{CNCO}_{2}$, y CNOM.

\section{$3.1 \mathrm{CNI}$}

El CNI indica la cantidad monetaria que debe invertirse en una central para generar un MWh de energía eléctrica. Se obtiene del cociente del valor futuro de los costos de inversión en la construcción de la central, entre el valor presente de la generación neta anual de energía eléctrica de la central, durante su vida útil. Los parámetros de entrada y la metodología para calcular el CNI son publicados por IEA (2015). ${ }^{11}$ La Tabla 1 muestra dichos parámetros para las tres tecnologías de interés.

Tabla 1. Parámetros de entrada para calcular el CNI

\begin{tabular}{|c|c|c|c|c|}
\hline Concepto & Unidades & TC & CC & CN \\
\hline Capacidad & MW & 1,131 & 551 & 1,425 \\
\hline Factor de planta & $\%$ & $85 \%$ & $85 \%$ & $85 \%$ \\
\hline Usos propios & $\%$ & $10.6 \%$ & $3.0 \%$ & $3.5 \%$ \\
\hline Costo unitario de inversión & Dólares $/ \mathrm{kW}$ & 1,987 & 979 & 4,168 \\
\hline Vida económica & años & 40 & 30 & 60 \\
\hline Tasa anual de descuento $^{13}$ & $\%$ & $4.93 \%$ & $4.93 \%$ & $4.93 \%$ \\
\hline
\end{tabular}

Fuente: Elaboración propia con datos de IEA (2015) y CFE (2014)

Debido a que la construcción de una central de carga base toma más de un año, se requiere contar con un programa de inversión. El programa de inversión de las diferentes centrales se encuentra detallado en CFE (2014). La información correspondiente se presenta a continuación.

Tabla 2. Programa de Inversión de las Centrales

\begin{tabular}{|c|c|c|c|}
\hline & TC & CC & CN \\
\hline Año & \%Inversión & \%Inversión & \%Inversión \\
\hline-5 & & & $3.5 \%$ \\
-4 & $11.6 \%$ & & $16.1 \%$ \\
-3 & $58.8 \%$ & $9.4 \%$ & $41.7 \%$ \\
-2 & $25.7 \%$ & $72.1 \%$ & $30.7 \%$ \\
\hline
\end{tabular}

\footnotetext{
${ }^{11}$ Una descripción sintetizada del cálculo del CTNG, así como de sus componentes, se encuentra en Gómez-Ríos (2008).
} 


\begin{tabular}{|c|c|c|c|}
\hline & TC & CC & CN \\
\hline Año & \%Inversión & \%Inversión & \%Inversión \\
\hline-1 & $3.9 \%$ & $18.5 \%$ & $8.0 \%$ \\
0 & $100.0 \%$ & $100.0 \%$ & $100.0 \%$ \\
\hline
\end{tabular}

Fuente: Elaboración propia con datos de CFE (2014).

Partiendo de lo anterior, se procede a aplicar la metodología para calcular el CNI. Los datos correspondientes se presentan a continuación.

Tabla 3. Costo Nivelado de Inversión (CNI)

\begin{tabular}{|l|l|c|c|c|}
\hline \multicolumn{1}{|c|}{ Concepto } & \multicolumn{1}{c|}{ Unidades } & TC & CC & CN \\
\hline Inversión sin intereses & Millones de dólares & $2,246.9$ & 539.4 & $5,969.4$ \\
Inversión con intereses & Millones de dólares & $2,569.9$ & 591.5 & $6,790.6$ \\
Generación bruta anual & MWh & $8,421,426$ & $4,102,746$ & $10,610,550$ \\
Generación neta anual & MWh & $7,528,755$ & $3,979,664$ & $10,239,181$ \\
CNI & Dólares/MWh & $\mathbf{\$ 1 8 . 7 7}$ & $\mathbf{\$ 9 . 1 4}$ & $\mathbf{\$ 3 2 . 9 8}$ \\
\hline
\end{tabular}

\section{$3.2 \mathrm{CNC}$}

El CNC indica la cantidad monetaria que debe destinarse a la compra del combustible necesario para generar un MWh de energía eléctrica. Su cálculo se obtiene del cociente del valor presente del costo del combustible de la central, dividido por el valor presente de la generación neta anual de energía eléctrica de la central. Los parámetros de entrada requeridos para calcular el CNC son: 1) tasa de descuento; 2) eficiencia de la central, y; 3) precio de los combustibles.

El carbón es el combustible empleado por termoeléctrica de carbón. La central nuclear emplea uranio, mientras que la central de ciclo combinado emplea gas natural. Las proyecciones de los precios de los combustibles se toman de AEO (2017). Se estima que el precio del carbón pasará de $\$ 2.23$ dólares $/$ mmbtu en el 2017 a $\$ 4.83$ dólares $/ \mathrm{mmbtu}$ en el año 2050. Por otro lado, se estima que el precio del uranio pasará de $\$ 0.61$ dólares $/ \mathrm{mmbtu}$ en 2017 a $\$ 2.90$ dólares $/ \mathrm{mmbtu}$ para 2050. Finalmente, se proyecta un cambio en el precio del gas natural de $\$ 3.61$ dólares/mmbtu en 2017 a $\$ 12.41$ dólares $/$ mmbtu para 2050. La Gráfica 4 presenta las tendencias de los precios de los combustibles.

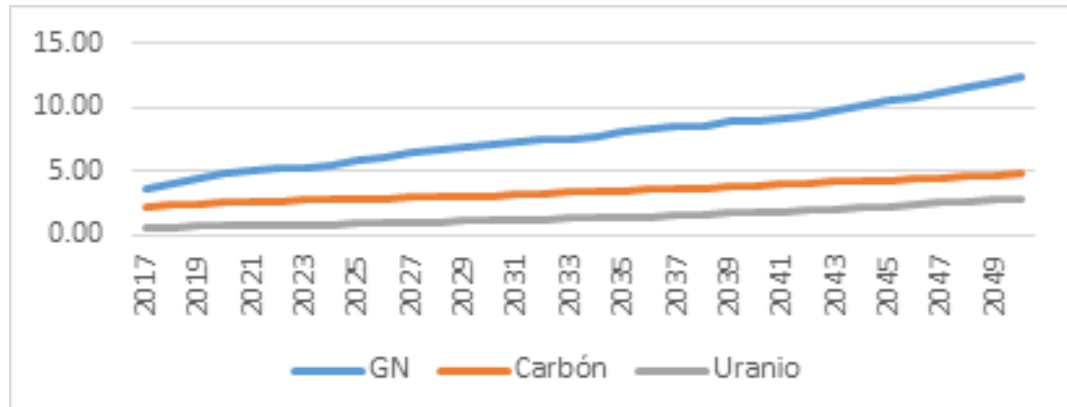

Gráfica 1. Proyecciones del Precio de los Combustibles (dólares/mmbtu) Fuente: Elaboración propia con datos de AEO (2017).

El CNC de la termoeléctrica de carbón se calcula tomando la media de las proyecciones de los precios del carbón, que asciende a $\$ 3.44$ dólares $/ \mathrm{mmbtu}$ (incluye 1 dólar de transporte).

Para que el uranio pueda ser empleado en la central nuclear, requiere someterse a un proceso de enriquecimiento y fabricación. De este modo, el CNC de la central nuclear es de $\$ 9.33$ dólares/MWh y se compone de dos partes: El costo del combustible es de $\$ 7.0$ dólares/MWh, mientras que $\$ 2.33$ dólares/MWh se destinan al tratamiento y disposición 
REMEF (The Mexican Journal of Economics and Finance) Costo de generación eléctrica incorporando externalidades ambientales:

del combustible gastado (NEA/IEA (2015)). Es de destacar que el CNC de la central nuclear incluye los costos de tratamiento y disposición del combustible gastado (Ramírez et al., 2006).

El CNC de la central de ciclo combinado considera la proyección de los precios del gas natural.

Para calcular el CNC se considera una tasa de descuento de $4.93 \%$. Debido a que la producción de electricidad por parte del ciclo combinado obedece a la demanda, dependiendo de la hora del día, no todas las plantas operan a plena carga. Lo que afecta la eficiencia del equipo. Por tal, se asume que la eficiencia de la central de ciclo combinado de $46.92 \%{ }^{14}$ La central nuclear y la termoeléctrica de carbón operan a plena carga las 24 horas del día. Por lo que no se incluye su nivel de eficiencia. De esta manera, el CNC de: a) la termoeléctrica de carbón es $\$ 37.37$; b) la central de ciclo combinado es de $\$ 48.35$, y; c) central nuclear es de $\$ 9.33$. El CNC se expresa en dólares/MWh.

\section{3 $\mathrm{CNCO}_{2}$ (Externalidades)}

El $\mathrm{CNCO}_{2}$ captura el costo de las emisiones de $\mathrm{CO}_{2}$ por cada MWh generado. Los parámetros que se necesitan para calcular el $\mathrm{CNCO}_{2}$ son la tasa de descuento y el costo del $\mathrm{CO}_{2}$. La termoeléctrica de carbón y la central de ciclo combinado generan respectivamente 730.0 y 376.9 kilogramos de $\mathrm{CO}_{2}$ por MWh generado (CFE, 2014). El costo por tonelada de $\mathrm{CO}_{2}$ emitidas al ambiente se estima en $\$ 36.35$ dólares. ${ }^{15}$ Por tal, la termoeléctrica de carbón tiene un costo nivelado por emisiones de $\mathrm{CO}_{2}$ de $\$ 29.68$ dólares/MWh, mientras que el costo correspondiente de la central de ciclo combinado es de $\$ 14.12$ dólares/MWh.

Es de destacar que, las centrales nucleares no producen $\mathrm{CO}_{2}$ en su proceso de generación de electricidad.

\subsection{CNOM}

El CNOM indica la cantidad monetaria que tiene que destinarse a la central para cubrir los costos de la operación y mantenimiento por cada MWh generado. Se obtiene al dividir el valor presente de los costos de operación y mantenimiento entre el valor presente de la generación neta anual de energía eléctrica de la central. Su cálculo requiere de la tasa de descuento, así como de la capacidad de la central.

Para estimar el CNOM de cada una de las tres centrales de carga base, se empleó información de la IEA (2015). Se asume también la tasa de descuento de $4.93 \%$ y la eficiencia de la central de ciclo combinado de $46.92 \%$. Así, el CNOM de la termoeléctrica de carbón es de $\$ 12.44$ dólares/MWh, el del ciclo combinado asciende a $\$ 4.79$ dólares/MWh. Finalmente, la central nuclear tiene un CNOM de $\$ 11.40$ dólares/MWh.

Ahora se cuenta con todos los elementos que integran el CTNGE, mismo que se calculará en el siguiente apartado.

\subsection{CTNGE}

De acuerdo con la expresión (1), el cálculo del CTNGE de cada una de las tecnologías, utilizando el modelo determinístico, se obtiene al sumar los costos nivelados calculados previamente. La Tabla 5 resume los CTNGE de las diferentes tecnologías.

\footnotetext{
${ }^{14} \mathrm{La}$ eficiencia del ciclo combinado se estima en el apartado 3.1.2.

${ }^{15}$ Este valor se estimó con base a información de las proyecciones 2018-2028 realizadas por el ICF Consulting Canada (2017).
} 
Tabla 4. Costo Total Nivelado de Generación con Externalidades (Dólares/MWh)

\begin{tabular}{|l|c|c|c|c|c|c|}
\hline & \multicolumn{2}{|c|}{ TC } & \multicolumn{2}{c|}{ CC } & \multicolumn{2}{c|}{ CN } \\
\hline Costo Nivelado de Inversión & $\$ 18.77$ & $19.1 \%$ & $\$ 9.14$ & $12.0 \%$ & $\$ 32.98$ & $61.4 \%$ \\
\hline Costo Nivelado de Combustible & $\$ 37.37$ & $38.0 \%$ & $\$ 48.35$ & $63.3 \%$ & $\$ 9.33$ & $17.4 \%$ \\
\hline Costo Nivelado del $\mathrm{CO}_{2}$ & $\$ 29.68$ & $30.2 \%$ & $\$ 14.12$ & $18.5 \%$ & $\$ 0.0$ & $0.0 \%$ \\
\hline Costo Nivelado de Operación y Mtto. & $\$ 12.44$ & $12.7 \%$ & $\$ 4.79$ & $6.3 \%$ & $\$ 11.40$ & $21.2 \%$ \\
\hline CTNGE & $\$ \mathbf{9 8 . 2 6}$ & $\mathbf{1 0 0 . 0 \%}$ & $\$ \mathbf{7 6 . 4 0}$ & $\mathbf{1 0 0 . 0 \%}$ & $\$ \mathbf{5 3 . 7 1}$ & $\mathbf{1 0 0 . 0 \%}$ \\
\hline
\end{tabular}

Fuente: Elaboración propia con datos de IEA (2015), AEO (2017), CFE (2014) e ICF

Consulting Canada (2017).

Es evidente que la central nuclear muestra el menor CTNGE, de \$53.71 dólares/MWh. Puede argumentarse que esto se debe, al menos en parte, a que la central nuclear no incurre en costos de $\mathrm{CO}_{2}$. No obstante, la central nuclear mostraría un CTNGE menor que el de las otras tecnologías si enfrentara los mismos costos de $\mathrm{CO}_{2}$. En tales casos, el CTNGE de la central nuclear sería de $\$ 83.39$ y $\$ 67.83$ dólares/MWh. Adicionalmente, los costos nivelados que integran el CTNGE no son fijos o inamovibles. Están sujetos a cambios de sus componentes (por ejemplo: la tasa de descuento, costo unitario de inversión, costo del $\mathrm{CO}_{2}$, eficiencia de la central, precio de los combustibles, etcétera). Por lo que no es posible asegurar que los CTNGE de las diferentes tecnologías se mantendrán en los montos obtenidos en esta sección.

A continuación, se analiza la participación de los costos nivelados dentro del CTNGE de cada una de las diferentes tecnologías analizadas. La gráfica 2 muestra dicha composición.

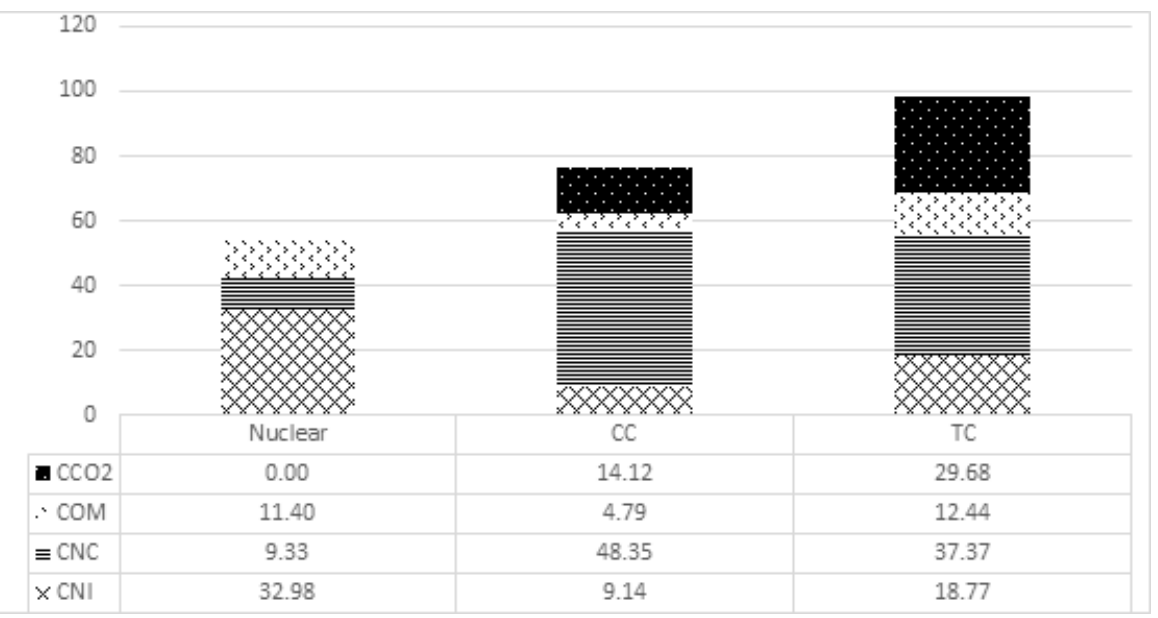

Gráfica 2. Participación de los costos nivelados dentro del CTNGE Fuente: Elaboración propia con datos de IEA (2015), AEO (2017), CFE (2014) e ICF Consulting Canada (2017).

Partiendo de los resultados del modelo determinístico, el costo nivelado del combustible es el que más contribuye a los CTNGE de la termoeléctrica de carbón (38\%) y del ciclo combinado (63.3\%). Esto hace que los CTNGE de dichas tecnologías sean extremadamente dependientes del precio de los combustibles correspondientes: carbón y gas natural. Adicionalmente, el $\mathrm{CNCO}_{2}$ contribuye en segundo lugar a los CTNGE de la termoeléctrica de carbón $(30.2 \%)$ y del ciclo combinado $(18.5 \%)$. El hecho de los precios de los combustibles y del $\mathrm{CNCO}_{2}$ sean volátiles y cambien a través del tiempo implica que, el CTNGE también será variable a través del tiempo. Por tal, no se puede asegurar que los CTNGE de estas tecnologías se mantendrán en los valores obtenidos.

Por otro lado, el CTNGE de la central nuclear depende mayormente del costo nivelado de inversión (61.4\%). Debido a que la construcción de una central nuclear toma alrededor de cinco años, el costo nivelado de inversión está sujeto a cambios en la tasa de descuento 
y en el costo unitario de inversión. Lo que implica que el CTNGE cambiará cuando cambie la tasa de descuento. Por tal, tampoco se puede asegurar que el CTNGE de la central nuclear se mantendrá en el valor obtenido.

Es evidente que el cálculo del CTNGE de manera determinística presenta varias limitaciones (Roques, 2006 y Karkhov, 2002). Éstas quedan cubiertas, al menos parcialmente, al emplear el método estocástico (Hrafnkelsson, 2016 y Khindanova, 2013).

Partiendo de lo anterior, en la siguiente sección se calculan los CTNGE utilizando un método estocástico. Particularmente, se emplea simulación Monte Carlo para describir las variaciones de los componentes que tienen mayor impacto en el CTNGE de cada una de las diferentes tecnologías.

\section{Estimación del CTNGE utilizando el Método estocástico}

En esta sección se emplea el Método de simulación Monte Carlo para generar variables aleatorias cuyas densidades de probabilidad describen los CTNGE de las tres tecnologías. Particularmente, se emplea el método de transformada inversa (ver Ross, 1999, para mayor detalle).

Se comienza por generan variables aleatorias cuyas densidades de probabilidad describan respectivamente a los siguientes parámetros: 1) costo unitario de inversión de las tres tecnologías (IEA, 2015); 2) nivel de eficiencia de las centrales de ciclo combinado (CENACE, 2010-2012, Información estadística); 3) precio de las emisiones de $\mathrm{CO}_{2}$ en las tecnologías fósiles (ICF Consulting Canada, 2017), y; 4) tasa de descuento en las tres tecnologías (OCDE, 2017).

Con la finalidad de aislar el efecto que tienen cada uno de los parámetros descritos en el párrafo anterior sobre el CTNGE, se realizan los siguientes supuestos: i) no hay variaciones estocásticas en el precio de los combustibles. Para ello, su asume que el carbón y el uranio enriquecido tendrán un precio constante de 3,44dlares/mmbtuy9.33 usd/MWh respectivamente. Se supone también que el precio del gas natural seguirá las proyecciones descritas en AEO (2017) para el periodo 2017-2050.; ii) La central nuclear y la termoeléctrica de carbón operan a plena carga las 24 horas del día. Por lo que no se incluye su nivel de eficiencia. Debido a que la producción de electricidad por parte del ciclo combinado obedece a la demanda, dependiendo de la hora del día, no todas las plantas operan a plena carga. Lo que afecta la eficiencia del equipo.; iii) El CNOM de cada central no depende del nivel de generación de electricidad. Por tal, se consideran como costos fijos debido a que las variaciones que pudieran presentar son mínimas. Se asume entonces que el CNOM de cada tecnología tomará los valores obtenidos en la sección anterior donde se empleó el método determinístico.

La simulación Monte Carlo se lleva a cabo realizando 100,000 iteraciones. Para ello, se emplea el software especializado @Risk versión 7.5 de la empresa Palisade.

\subsection{Variables Aleatorias que Describen los Parámetros de Entrada}

Formalmente, en esta sección se emplea simulación Monte Carlo para estiman las variables aleatorias $X_{1}^{j}, X_{2}^{j}, X_{3}^{j}, X_{4}^{j}$, correspondientes a los parámetros de entrada para la tecnología $j$, con $j=t c, c c, c n$. Las densidades de probabilidad de dichas variables aleatorias son $f_{i}^{j}\left(x_{i}^{j}\right)$ para $i=1,2,3,4$ y $j=t c, c c, c n$.

\subsubsection{Costo Unitario de Inversión}

Se dispone de pocos datos sobre los costos unitarios de inversión de las tres tecnologías. Para cada caso, solo se cuenta con los valores máximo, mínimo y la media (IEA, 2015). Por tal, se asume que las variables aleatorias de los costos unitarios de inversión de las tres tecnologías se distribuyen de acuerdo con densidades de probabilidad triangulares ${ }^{16}$

\footnotetext{
${ }^{16}$ Ver el capítulo 44 de Forbes et al. (2011) para los detalles de la distribución Triangular.
} 
cuyas características principales se listan en la siguiente tabla:

Tabla 5. Costo Nivelado de Inversión (Dólares/kWe)

\begin{tabular}{|l|c|c|c|}
\hline & TC & CC & CN \\
\hline Valor máximo & $\$ 3,067$ & $\$ 1,289$ & $\$ 6,215$ \\
\hline Valor mínimo & $\$ 813$ & $\$ 627$ & $\$ 1,807$ \\
\hline Media & $\$ 1,987$ & $\$ 979$ & $\$ 4,168$ \\
\hline Desviación estándar & $\$ 461$ & $\$ 136$ & $\$ 907$ \\
\hline
\end{tabular}

\subsubsection{Eficiencia de las Centrales de Ciclo Combinado}

La información estadística del nivel de eficiencia de las centrales de ciclo combinado en México, para el período 2010-2012, se toma del CENACE. La variable aleatoria que mejor describe la eficiencia de las centrales de ciclo combinado tiene una densidad de probabilidad Logística, ${ }^{17}$ que se encuentra en un rango, con valor mínimo de $39.413 \%$ y máximo de $51.277 \%$, siendo el valor medio de $46.929 \%$ y la desviación estándar de $2.517 \%$.

\subsubsection{El Precio de las Emisiones de $\mathrm{CO}_{2}$}

La variable aleatoria que mejor describe el precio de las emisiones de $\mathrm{CO}_{2}$, considerando el período 2018-2028, ${ }^{18}$ tiene una densidad de probabilidad Triangular, con valor máximo de $\$ 68.32$ dólares/ton $\mathrm{CO}_{2}$, valor mínimo de $\$ 16.89$ dólares/ton $\mathrm{CO}_{2}$, media de $\$ 36.347$ dólares/ton $\mathrm{CO}_{2}$ y desviación estándar de $\$ 11.393$ dólares/ton $\mathrm{CO}_{2}$.

\subsubsection{La Tasa de Descuento}

La Tasa de Referencia de Interés Comercial (CIRR, por sus siglas en inglés) se toma como una aproximación de la tasa de descuento (OCDE, 2017). La CIRR es la tasa de descuento que se aplica en los países miembros de la OCDE, incluido México, para financiar proyectos de largo plazo. Se considera una serie mensual para el período de enero de 2009 a diciembre de 2017. También se ha incluido un factor de ajuste que incluye el riesgo-país de México, que de acuerdo con la SHCP (2017), para el 29 de diciembre de 2017, se situaba en 189 punto-base. La serie histórica de la CIRR se muestra en la Gráfica 3.

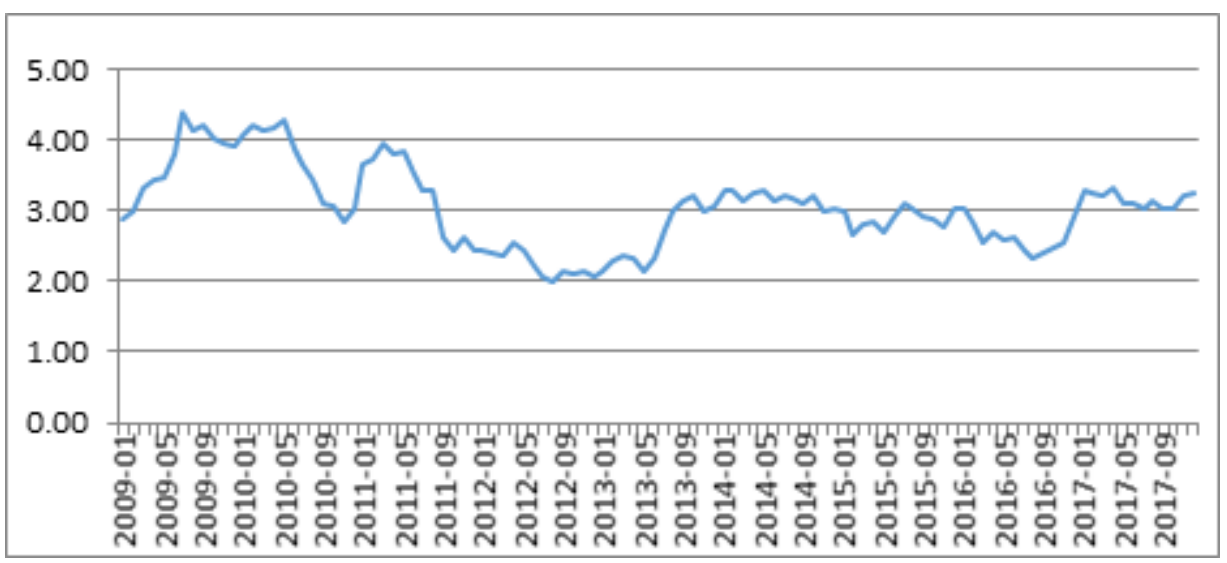

Gráfica 3. Comportamiento histórico de la CIRR Fuente: OCDE (2017).

\footnotetext{
${ }^{17}$ Ver el capítulo 28 de Forbes et al. (2011) para los detalles de la distribución Logística.

${ }^{18}$ Los datos se toman del ICF Consulting Canada (2017).
} 
Partiendo de este conjunto de información, la variable aleatoria que mejor describe a la CIRR, tiene una densidad de probabilidad Weilbull, con valor máximo de $4.37 \%$, mínimo de $1.98 \%$, media de $3.0353 \%$ y una desviación estándar de $0.5785 \%$.

Ahora se cuenta con las variables aleatorias de los parámetros de entrada. Partiendo de ellos, en la siguiente subsección se emplea nuevamente la simulación Monte Carlo para estimar las variables aleatorias que mejor describen a los CTNGE de las diferentes tecnologías.

\subsection{CTNGE - Método estocástico}

En este apartado se emplea simulación Monte Carlo para generar las variables aleatorias $X_{c n}, X_{c c}$ y $X_{t c}$, correspondientes a los CTNGE de las tres tecnologías. Las variables aleatorias de los CTNGE son funciones de las variables aleatorias de los parámetros de entrada. Esto es

$$
X_{j}=\pi_{j}\left(X_{1}^{j}, X_{2}^{j}, X_{3}^{j}, X_{4}^{j}\right) \quad \text { para } j=t c, c c, c n .
$$

Suponga que $X_{c n}$ toma valores en $x_{c n} \in[a, b]$, cuya densidad de probabilidad es $f$ y correspondiente distribución acumulada $F$. La variable aleatoria $X_{c c}$ toma valores en $x_{c c} \in[a, b]$. Su densidad de probabilidad es $g$ con la correspondiente distribución acumulada $G$. Finalmente, $X_{t c}$ toma valores en $x_{t c} \in[a, b]$, con densidad de probabilidad $h$ y correspondiente distribución acumulada $H$.

Nuevamente se emplea el software @Risk versión 7.5 de la empresa Palisade para realizar la simulación Monte Carlo. Para generar las variables aleatorias de los CTNGE se realizaron 100,000 iteraciones.

La gráfica 9 muestra las densidades de probabilidad de los CTNGE de las tres tecnologías. La tabla 10 presenta las principales características de dichas densidades de probabilidad.

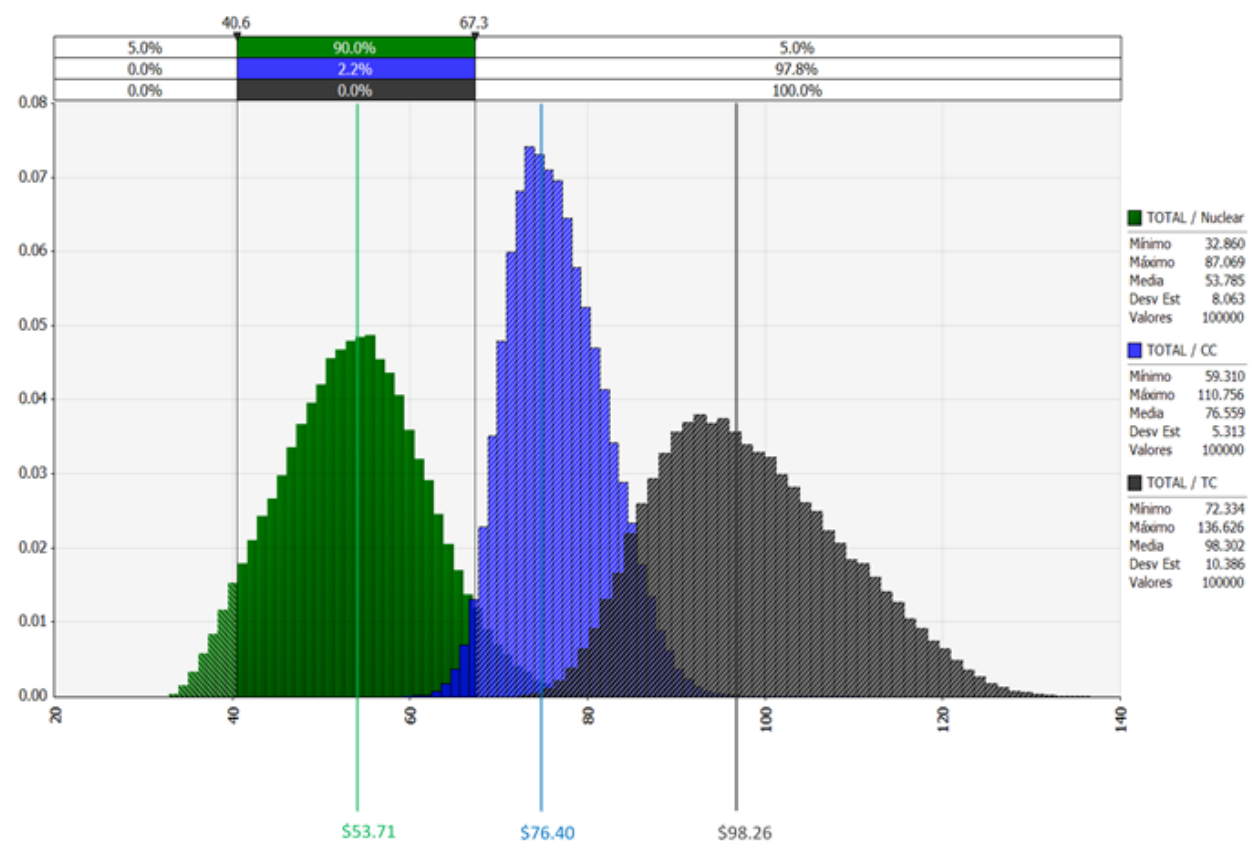

Gráfica 4. Densidades de probabilidad de los CTNGE de las tres tecnologías Fuente: Elaboración propia con datos obtenidos de la simulación. 
Tabla 6. CTNGE (Dólares/MWh)

\begin{tabular}{|l|c|c|c|}
\hline & TC & CC & CN \\
\hline Valor máximo & $\$ 136.626$ & $\$ 110.756$ & $\$ 87.069$ \\
\hline Valor mínimo & $\$ 72.334$ & $\$ 59.310$ & $\$ 32.860$ \\
\hline Media & $\$ 98.302$ & $\$ 76.559$ & $\$ 53.785$ \\
\hline Desviación estándar & $\$ 10.386$ & $\$ 5.313$ & $\$ 8.063$ \\
\hline
\end{tabular}

Fuente: Elaboración propia con datos obtenidos de la simulación.

De los datos anteriores se destacan dos aspectos. Primero, las medias de las densidades de probabilidad de los CTNGE son muy parecidas a los CTNGE obtenidos con el método determinístico, descritos en la tabla 5.

En segundo lugar, es evidente que el CTNGE de la termoeléctrica de carbón, además de tener la mayor media, es el más riesgoso al presentar la mayor desviación estándar. Por otro lado, la media del CTNGE de la central nuclear es menor a la media del CTNGE del ciclo combinado. Sin embargo, la central nuclear muestra un CTNGE más riesgoso que el correspondiente CTNGE del ciclo combinado.

En el siguiente apartado se realiza una comparación más detallada entre los CTNGE de las tres tecnologías.

\subsection{Comparando los CTNGE de las Diferentes Tecnologías}

Aparentemente, la central nuclear tiene los menores CTNGE. Por tal, para una primera comparación, se toma como referencia el CTNGE de la central nuclear. Se considera un intervalo en el que se deja fuera a los primeros y los últimos cinco percentiles. Así, existe una probabilidad de 0.90 (90\%) de que el CTNGE de la central nuclear se encuentre en el intervalo $r=[40,6,67,3] .{ }^{19}$ La probabilidad de que el CTNGE del ciclo combinado se encuentre en el intervalo $\mathrm{r}$ es de $0.022(2.2 \%)$. Finalmente, existe una probabilidad de 0 $(0 \%)$ de que el CTNGE de la termoeléctrica de carbón se encuentre en el intervalo $r$. Este análisis muestra que el ciclo combinado exhibe mayores CTNGE que la central nuclear. Además, la termoeléctrica de carbón es la tecnología con los mayores CTNGE.

Para una comparación más detallada entre los CTNGE de las diferentes tecnologías, se emplea el concepto de dominancia estocástica de primer orden. Para ello, partiendo de los datos descritos en la tabla 10, primero se supone que las densidades de probabilidad de los CTNGE de las tres tecnologías toman valores en el intervalo $D=[32,860,136,626]$. Por ejemplo, para el CTNGE de la central nuclear, se cumple que $f>0$ para $x_{c n} \in$ $(32,860,87,069)$ y $f=0$ para $x_{c n} \in[87,069,136,626]$.

Posteriormente, se verifica la dominancia estocástica de primer orden entre pares de tecnologías. Para ello, se comienza comparando al CTNGE de la central nuclear, cuya distribución acumulada (DA) es F, con el CTNGE del ciclo combinado, cuya DA es G. Finalmente se extiende el resultado a la DA del CTNGE de la termoeléctrica de carbón, $\mathrm{H}$.

Formalmente, la Proposición G. D. 1. del libro de Mas-Colell et al. (1995) (pp. 195) se adapta al contexto del CTNGE, quedando como sigue: la DA del CTNGE del ciclo combinado, $G(\bullet)$, muestra dominancia estocástica de primer orden sobre la DA de la central nuclear, $F(\bullet)$, si y solo si $G(x)<F(x)$ para cada x. En otras palabras, se dice que "la función $G$ muestra dominancia estocástica de primer orden sobre la función $F$ si la DA $G$ produce, sin ambigüedades, CTNGE mayores que la DA F".

Partiendo de la anterior, para las DA de los CTNGE de las tres tecnologías, resulta evidente que $H(x)<G(x)<F(x)$ para cada $x$. Es decir, la DA del CTNGE de la termoeléctrica de carbón muestra dominancia estocástica de primer orden sobre la DA del CTNGE de ciclo combinado y la central nuclear. En otras palabras, la termoeléctrica de carbón produce, sin ambigüedades, los mayores CTNGE. En segundo lugar, se encuentran

${ }^{19}$ Valores expresados en dólares/MWh. 
los CTNGE del ciclo combinado. Siendo la central nuclear la tecnología que produce los menores CTNGE.

Es de destacar que las densidades de probabilidad y las correspondientes DA de los CTNGE de las diferentes tecnologías no son definitivas o inamovibles. Pueden variar en forma o desplazarse si se modifica la distribución de probabilidad de alguno de los parámetros de entrada. Es decir, las distribuciones de los CTNGE del ciclo combinado y la termoeléctrica de carbón podrían desplazarse, por ejemplo, hacia la izquierda si el precio del $\mathrm{CO}_{2}$ disminuye. Así, los CTNGE de dichas tecnologías serían competitivos con el correspondiente CTNGE de la central nuclear. Es entonces necesario verificar el grado en que los diferentes parámetros de entrada afectan a las densidades de probabilidad de los CTNGE de las diferentes tecnologías. Con esta finalidad, en la siguiente sección se realiza el análisis de sensibilidad de Tornado.

\subsection{Análisis de Tornado}

El análisis de tornado se emplea para identificar que tan sensible es el CTNGE ante cambios en los parámetros de entrada. Es decir, el análisis de tornado ayuda a identificar el grado en el que los parámetros de entrada contribuyen a la incertidumbre del CTNGE.

El diagrama de tornado resume el impacto de cada parámetro de entrada. Típicamente este análisis asume que dichos parámetros son estadísticamente independientes. Para que la gráfica sea impactante, los parámetros se ordenan ubicando en la parte superior al que tiene mayor impacto y en la parte inferior al que tiene menor impacto. Así, la gráfica tiene la forma de un tornado. De este modo, el inversionista puede identificar fácilmente los parámetros de entrada en los que debe poner mayor atención.

El análisis de tornado requiere definir un rango de prueba. Para el presente análisis se toma el rango del percentil $5 \%$ al $95 \%{ }^{20}$

\subsubsection{Termoeléctrica de Carbón}

Para este caso se considera la variación de tres parámetros de entrada: 1) el costo unitario de inversión; 2) el precio de las emisiones de $\mathrm{CO}_{2}$, y; 3) la tasa de descuento. La Grafica 11 presenta el diagrama de tornado correspondiente.

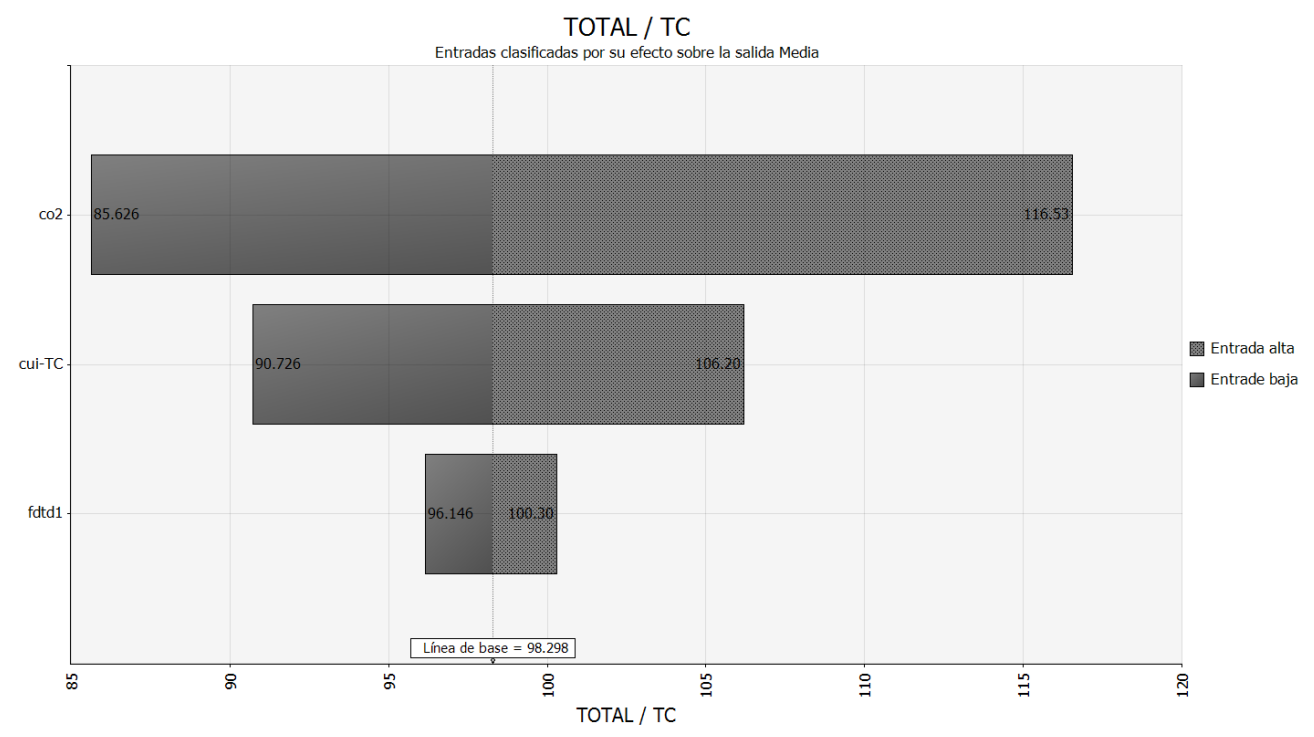

Gráfica 5. Análisis de Tornado - Termoeléctrica de Carbón

Fuente: Elaboración propia con datos simulados.

\footnotetext{
${ }^{20}$ Ver Eschenbach (2006) para mayor detalle sobre el diagrama tornado.
} 
Los parámetros que mayor impacto tienen sobre el CTNGE son el precio de las emisiones de $\mathrm{CO}_{2}$ y el costo unitario de inversión. Por un lado, si hay una variación al alza en el precio de las emisiones de $\mathrm{CO}_{2}$, el CTNGE pasa de $\$ 98.30$ a $\$ 116.53$ dólares/MWh. Por otro lado, si el costo unitario de inversión tiene un movimiento al alza, el CTNGE pasa de $\$ 98.30$ a $\$ 106.20$ dólares/MWh.

\subsubsection{Ciclo Combinado}

Para el ciclo combinado se considera la variación de cuatro parámetros de entrada: 1) el costo unitario de inversión; 2) la eficiencia de las centrales; 3) el precio de las emisiones de $\mathrm{CO}_{2}$, y; 4) la tasa de descuento. En la Grafica 12 se muestra el diagrama de tornado correspondiente

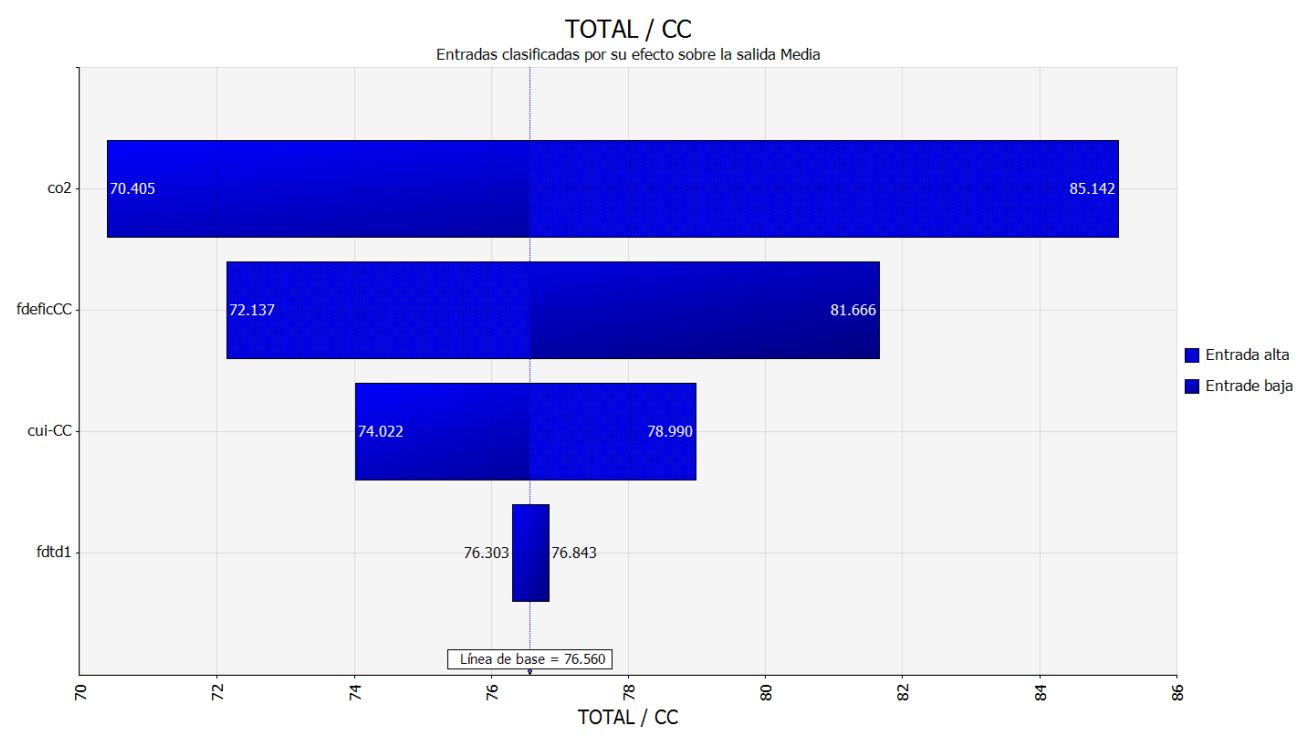

Gráfica 6. Análisis de Tornado - Ciclo Combinado

Fuente: Elaboración propia con datos obtenidos de la simulación.

De la Gráfica 6 es directo verificar que el CTNGE del ciclo combinado es más sensible a cambios en el precio de las emisiones de $\mathrm{CO}_{2}$. Si hay una variación al alza, el CTNGE pasa de $\$ 76.56$ a $\$ 85.14$ dólares/MWh. El CTNGE del ciclo combinado es también sensible ante cambios en la eficiencia de la central. Si aumenta, el CTNGE pasa de $\$ 76.56$ a $\$ 72.14$ dólares/MWh.

\subsubsection{Central Nuclear}

Finalmente, el diagrama de tornado para la central nuclear, que se presenta en la Gráfica 13, considera la variación de dos parámetros de entrada: 1) el costo unitario de inversión, y; 2) la tasa de descuento. 


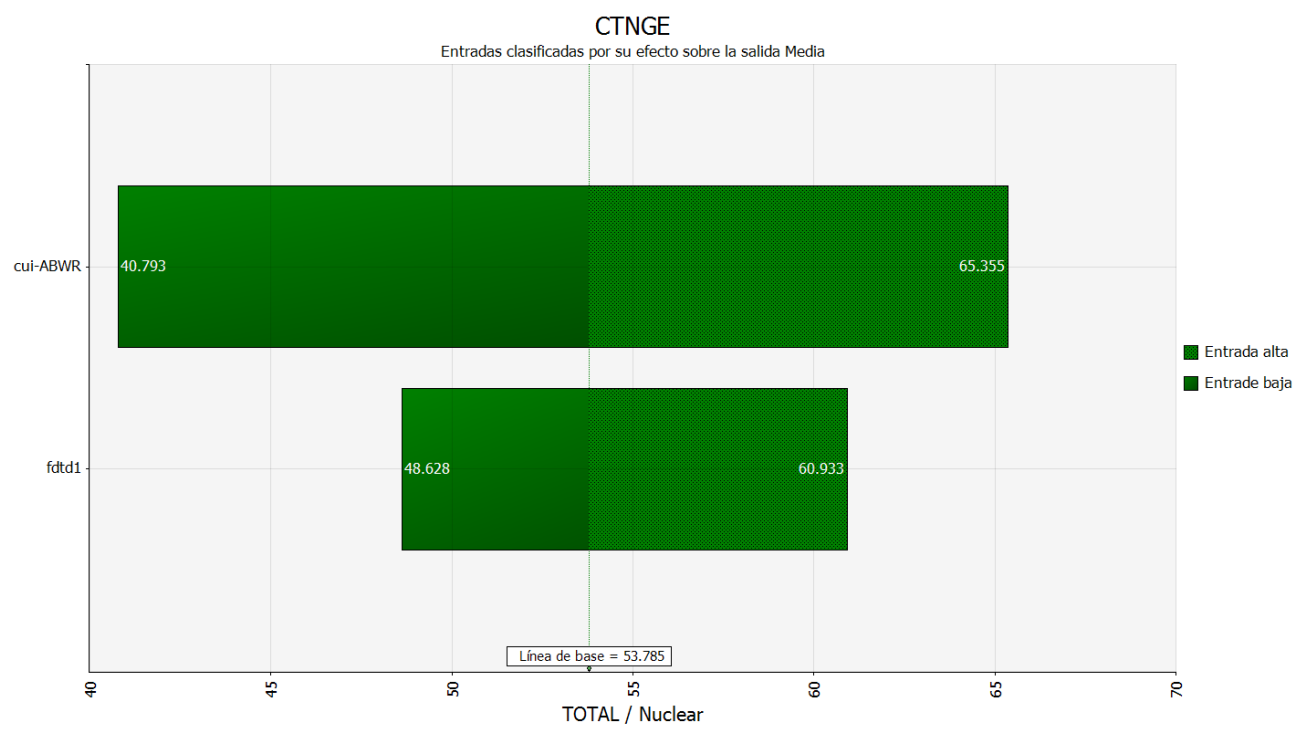

Gráfica 7. Análisis de Tornado - Central Nuclear

Fuente: Elaboración propia con datos obtenidos de la simulación.

Al ser una tecnología intensiva en capital, el parámetro que mayor impacto tiene sobre el CTNGE es el costo unitario de inversión, ya que, si hay una variación al alza, el CTNGE pasa de $\$ 53.785$ a $\$ 65.355$ dólares/MWh. El impacto de la tasa de descuento en el CTNGE, siendo menor, es muy significativo. Un incremento en la misma hace que el CTNGE pase de $\$ 53.785$ a $\$ 60.933$ dólares/MWh.

Debido a las variaciones a las que están sujetas los CTNGE de las diferentes tecnologías, sería adecuado enfocarse en un portafolio que incluya más de una tecnología, en lugar de centrarse en solo una tecnología. En la siguiente sección se especifican las condiciones bajo las cuales un portafolio con más de una tecnología es viable.

\section{Mezcla Óptima de Tecnologías de Carga Base}

Partiendo de los resultados obtenidos en las secciones anteriores, es evidente que, sin ambigüedades, la central nuclear tiene los menores CTNGE. Dado este hecho, se podría sugerir que la generación de energía eléctrica de un país debería de darse mayormente a través de centrales nucleares. No obstante, esta implicación presenta tres inconvenientes: 1) no existe ninguna garantía de que los CTNGE de la central nuclear, siendo ahora los menores, se mantendrán en ese nivel. Pueden aumentar si las variaciones de algún componente subyacente así lo determinan.; 2) a ningún país o economía le es viable depender de una sola tecnología para abastecer su demanda por electricidad, y; 3 ) la energía nuclear presenta gran oposición social.

Por consiguiente, en esta sección se emplea la teoría de portafolio de Markowitz (1952) para encontrar la mezcla de tecnologías de carga base que tenga el CTNGE menos riesgoso y con menor media. Como es usual, la medida de riesgo que se emplea es la desviación estándar (DE).

Formalmente se tiene lo siguiente: suponga se dispone de un monto de capital, que se normaliza a . El capital se puede destinar en su totalidad a una sola tecnología o dividirlo entre más de una tecnología. Sea la variable aleatoria que denota el CTNGE del portafolio que incluye las tres tecnologías. A partir de ahora se le denomina CTNGE-portafolio y se define como sigue:

$$
Y=\alpha_{1} X_{c n}+\alpha_{2} X_{c c}+\alpha_{3} X_{t c}, \quad \text { con } \quad \alpha_{1}+\alpha_{2}+\alpha_{3}=1 .
$$

Donde $\alpha_{i}$ denota la cantidad relativa asignada a la tecnología $i=1,2,3$. 
Para simplificar el análisis, sin pérdida de generalidad, se considera el caso de un portafolio con solo dos tecnologías. Lo que permitirá, al comparar entre pares de tecnologías, encontrar el mejor portafolio, el que muestre CTNGE con menor media y menor DE.

Considere dos diferentes tecnologías de generación de electricidad: 1 y 2 . Sea $0 \leq \alpha \leq 1$. Si el monto de capital se normaliza a 1, la cantidad relativa asignada a la tecnología 1 se describe por $\alpha$, mientras que la cantidad relativa asignada a la tecnología 2 queda descrita por $1-\alpha$. Sea Y la variable aleatoria que denota el CTNGE de portafolio que incluye dos tecnologías. Se denomina CTNGE-portafolio y se define como sigue:

$$
Y=\alpha X_{1}+(1-\alpha) X_{2}
$$

El siguiente resultado brinda las herramientas de análisis necesarias:

Lema 1. [basado en el teorema 4.14 (Freund et al., 2000 (pp. 158))]: Sea $X_{1}$ la variable aleatoria que denota el CTNGE de una tecnología de generación de electricidad con media $\mu_{1}$ y varianza $\sigma_{1}^{2}$. Sea $X_{2}$ la variable aleatoria que denota el CTNGE de una segunda tecnología de generación de electricidad con media $\mu_{2}$ y varianza $\sigma_{2}^{2}$. Sea la variable aleatoria $Y=\alpha X_{1}+(1-\alpha) X_{2}$, con $0 \leq \alpha \leq 1$. Entonces,

$$
\begin{gathered}
E(Y)=\alpha E\left(X_{1}\right)+(1-\alpha) E\left(X_{2}\right), \\
\mu_{Y}=\alpha \mu_{1}+(1-\alpha) \mu_{2} . \\
\mathrm{y} \\
\operatorname{Var}(Y)=E\left[\left(Y-\mu_{Y}\right)^{2}\right], \\
\sigma_{Y}^{2}=\alpha^{2} \sigma_{1}^{2}+(1-\alpha)^{2} \sigma_{2}^{2}+2 \alpha(1-\alpha) \sigma_{1,2} .
\end{gathered}
$$

donde $\sigma_{1,2}=E\left[\left(X_{1}-\mu_{1}\right)\left(X_{2}-\mu_{2}\right)\right]$ es la covarianza de los CTNGE de las dos tecnologías. ${ }^{21}$

Demostración: ver Freund et al. (2000).

El Lema 1 brinda dos resultados importantes. El primero se refiere a que la media del CTNGE-portafolio se encuentra entre las medias de los CTNGE de las tecnologías involucradas. El siguiente corolario describe este resultado.

Corolario 2. Si el CTNGE de la tecnología 2 tiene una mayor media, $\mu_{2}>\mu_{1}$, para $0 \leq \alpha \leq 1$ se cumple que $\mu_{2} \geq \mu_{Y} \geq \mu_{1}$. Lo contrario también se cumple: suponga que el CTNGE de la tecnología 1 tiene una mayor media, $\mu_{2}<\mu_{1}$, para $0 \leq \alpha \leq 1$ se cumple que $\mu_{2} \leq \mu_{Y} \leq \mu_{1}$.

El segundo resultado del Lema 1 se refiere al papel que tiene la covarianza en el cálculo de la varianza del CTNGE-portafolio. Si la covarianza de los CTNGE de las dos tecnologías es negativa, la varianza del CTNGE-portafolio será menor. Por otro lado, una covarianza positiva de los CTNGE de las dos tecnologías tiene como consecuencia una menor varianza del CTNGE-portafolio. En la sección 2.5 se analiza la composición de los CTNGE de las tres diferentes tecnologías de interés. Partiendo de ello, se espera que la covarianza entre los CTNGE de las diferentes tecnologías sea pequeña o nula. Por tal, para el presente análisis se considera el caso cuando no existe relación entre los CTNGE de las tecnologías implicadas. Es decir, cuando $\sigma_{1,2}=0$.

A partir de ahora, siguiendo la teoría de portafolio, utilizaremos la DE como medida de riesgo. Partiendo del Lema 1 , si $\sigma_{1,2}=0$, la DE del CTNGE-portafolio, Y, queda como sigue:

\footnotetext{
${ }^{21}$ En la teoría de portafolio es común expresar la covarianza en términos del coeficiente de correlación. No obstante, el no hacerlo de ese modo no altera el análisis.
} 
El riesgo del CTNGE-portafolio, expresado por su DE, es una función convexa de la cantidad relativa asignada a la tecnología 1, descrita por el parámetro $\alpha$. El siguiente resultado describe este hecho.

Proposición 3. Suponemos que el CTNGE de la tecnología 1 es más riesgoso que el CTNGE de la tecnología $2, \sigma_{1} \geq \sigma_{2}$. Para $\sigma_{1,2}=0$ y $0 \leq \alpha \leq 1$ se cumple que:

a) $\sigma_{Y}$ tiene su mínimo global en $\alpha^{*}=\sigma_{2}^{2}\left(\sigma_{1}^{2}+\sigma_{2}^{2}\right) \leq 12$. Dónde $\sigma_{Y}^{*}=$ $\sigma_{1}^{\sqrt{\alpha^{*}}<\sigma_{1} \sqrt{2}}$

b) Si $0 \leq \alpha \leq 2 \alpha^{*}$, entonces, $\sigma_{1} \sqrt{\alpha^{*}} \leq \sigma_{Y} \leq \sigma_{2}$

Demostración: ver apéndice.

Del Lema 1, el Corolario 2 y la Proposición 3, es evidente que el CTNGE-portafolio, así como su media y su DE quedan definidos por la cantidad relativa asignada a la tecnología más riesgosa, la tecnología 1 , dada por $\alpha$.

El primer resultado de la proposición 3 indica la cantidad relativa asignada a la tecnología con el CTNGE más riesgoso que garantiza el mínimo CTNGE-portafolio. Al destinar la cantidad relativa $\alpha^{*}$ de capital a la tecnología con el CTNGE más riesgoso, se garantiza que el CTNGE-portafolio será menos riesgoso que los CTNGE de las tecnologías 1 y 2. Esto es, $\sigma_{Y}^{*}<\sigma_{2} \leq \sigma_{1}$.

El segundo resultado garantiza que, para un intervalo de cantidades relativas asignadas a la tecnología con el CTNGE más riesgoso, el CTNGE-portafolio es aún menos riesgoso que el menos riesgoso de los CTNGE implicados. Este intervalo es donde se registra el "efecto portafolio" establecido en Awerbuch y Berger (2003): reducir el riesgo a través de la diversificación. Es evidente que el efecto portafolio se enfatiza cuando existe una covarianza negativa entre los CTNGE de las tecnologías implicadas en el portafolio. Es de destacar también que Awerbuch y Berger (2003) muestran el efecto portafolio a través de gráficas y simulaciones. En contraste, el presente trabajo encuentra formalmente el intervalo de cantidades relativas asignadas a la tecnología con el CTNGE más riesgoso, donde se registra el "efecto portafolio".

A continuación, se emplean los resultados antes descritos para analizar la factibilidad de los portafolios que incluyen pares de tecnologías. Para ello, se emplean los datos obtenidos en la sección 3.2, presentados en la tabla 10 .

\subsection{Termoeléctrica de Carbón y Ciclo Combinado}

En este portafolio, la termoeléctrica de carbón muestra el CTNGE más riesgoso con mayor media. Es decir, $\sigma_{t c}=10,386, \sigma_{c c}=5,313, \mu_{t c}=98,302$ y $\mu_{c c}=76,559$.

La Proposición 3 indica que si la termoeléctrica de carbón recibe la cantidad relativa $\alpha^{*}=\sigma_{2}^{2}\left(\sigma_{1}^{2}+\sigma_{2}^{2}\right)=28,228(107,87+28,228)=0,207$, el CTNGE-portafolio alcanza su menor valor que es $\sigma_{Y}^{*}=\sigma_{t c} \sqrt{\alpha^{*}}=10,386 \sqrt{0,207}=4,73$. La correspondiente media es $\mu_{Y}=79,308$. Las siguientes gráficas ilustran estos resultados. 


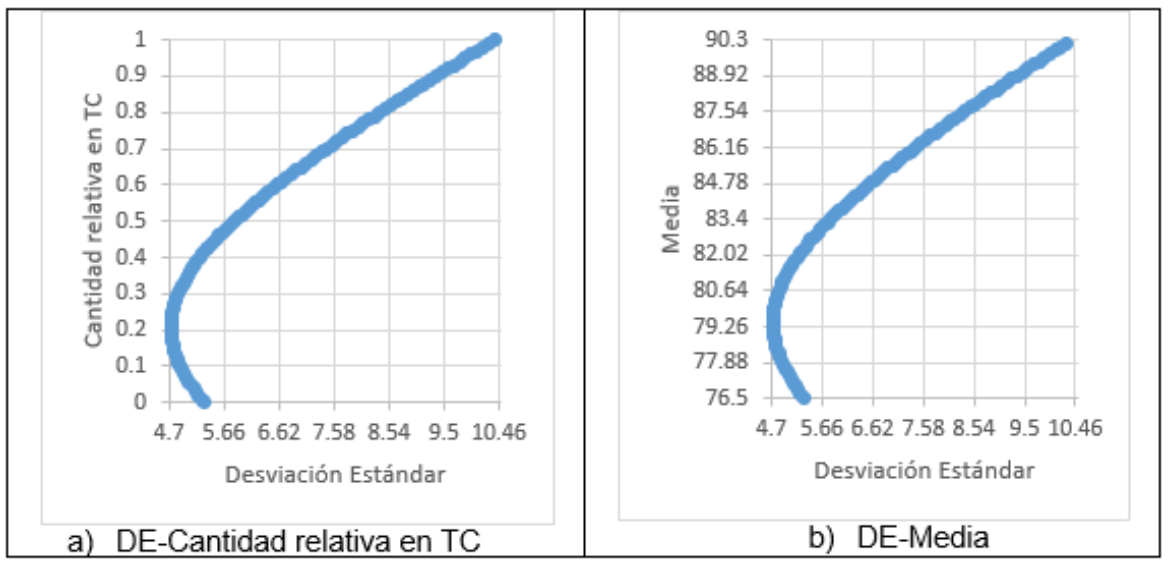

Gráfica 8. CTNGE-portafolio: Termoeléctrica de carbón y ciclo combinado Fuente: Elaboración propia con datos obtenidos de la simulación.

La parte a) de la gráfica 14 muestra la DE del CTNGE-portafolio, $\sigma_{Y}$, en relación con la cantidad relativa asignada a la termoeléctrica de carbón, $\alpha$. La parte b) muestra la relación entre la media y la DE del CTNGE-portafolio.

Los portafolios óptimos deben caer en la llamada "frontera eficiente". En el presente contexto, la "frontera eficiente" se tiene cuando la media del CTNGE del portafolio se puede reducir solo si se acepta un mayor riesgo. De acuerdo con la gráfica 14b, la "frontera eficiente" del presente portafolio se tiene cuando la DE del CTNGE se encuentra en el intervalo $5,313 \geq \sigma_{Y}^{*} \geq 4,73$, mientras que la media correspondiente se encuentra en el intervalo $76,559 \leq \mu_{Y} \leq 79,308$. De la gráfica 14a, es evidente que la "frontera eficiente" está definida cuando la cantidad relativa asignada a la termoeléctrica de carbón, $\alpha$, se encuentra en el intervalo factible, $0 \leq \alpha \leq 0,207$.

\subsection{Termoeléctrica de Carbón y Central Nuclear}

En este portafolio, la termoeléctrica de carbón muestra el CTNGE más riesgoso con mayor media. Es decir, $\sigma_{t c}=10,386, \sigma_{c n}=8,063, \mu_{t c}=98,302$ y $\mu_{c n}=53,785$.

Siguiendo a los resultados de la Proposición 3, la DE del CTNGE-portafolio alcanza su menor valor, que es $\sigma_{Y}^{*}=\sigma_{t c}^{\sqrt{\alpha^{*}}}=10,386 \sqrt{0,376}=6,369$, cuando la termoeléctrica de carbón recibe la cantidad relativa de $\alpha^{*}=\sigma_{2}^{2}\left(\sigma_{1}^{2}+\sigma_{2}^{2}\right)=65,012(107,87+65,012)=0,376$. La correspondiente media es $\mu_{Y}=67,661$. Las siguientes gráficas ilustran los resultados.

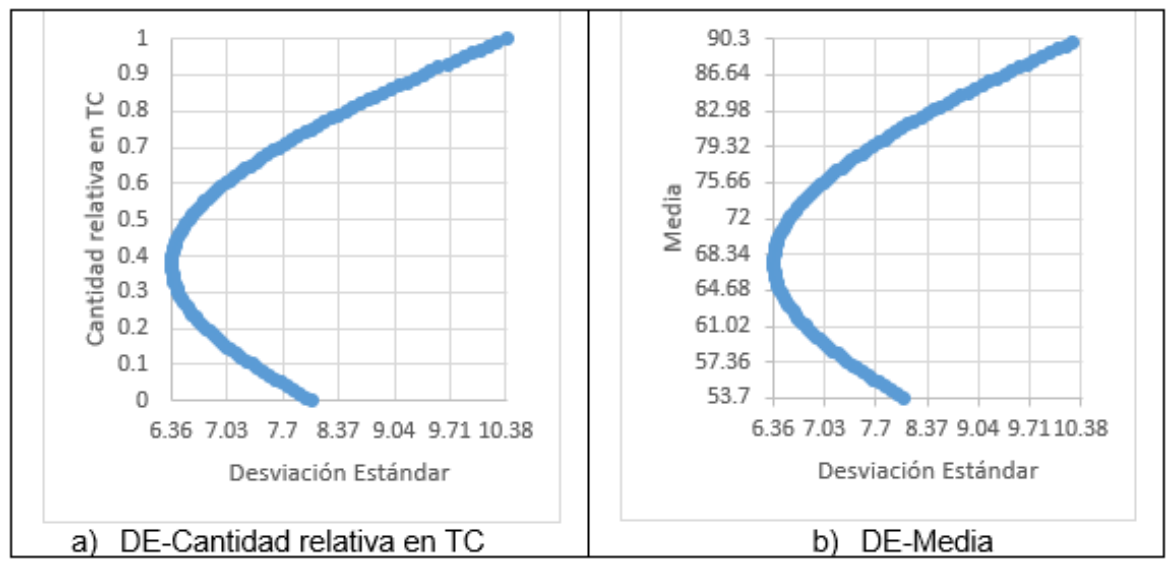

Gráfica 9. CTNGE-portafolio: Termoeléctrica de carbón y central nuclear Fuente: Elaboración propia con datos obtenidos de la simulación. 
La gráfica 15b indica que, la "frontera eficiente" del presente portafolio se tiene cuando la DE del CTNGE se encuentra en el intervalo $8,063 \geq \sigma_{Y}^{*} \geq 6,369$, mientras que la media correspondiente se encuentra en el intervalo $53,785 \leq \mu_{Y} \leq 67,661$. De la gráfica $15 \mathrm{a}$, es evidente que la "frontera eficiente" está definida cuando la cantidad relativa asignada a la termoeléctrica de carbón, $\alpha$, se encuentra en el intervalo factible, $0 \leq \alpha \leq 0,376$.

\subsection{Central Nuclear y Ciclo Combinado}

En los dos portafolios anteriores, una tecnología, la termoeléctrica de carbón, muestra el CTNGE más riesgoso y con mayor media. El presente portafolio es diferente, debido a que la central nuclear tiene el CTNGE más riesgoso, a la vez que presenta la menor media. Esto es, $\sigma_{c n}=8,063, \sigma_{c c}=5,313, \mu_{c n}=53,785$ y $\mu_{c c}=76,559$.

Si la central nuclear recibe la cantidad relativa de $\alpha^{*}=\sigma_{2}^{2}\left(\sigma_{1}^{2}+\sigma_{2}^{2}\right)=28,228(65,012+28,228)=$ 0,303, la DE del CTNGE-portafolio alcanza su menor valor que es $\sigma_{Y}^{*}=\sigma_{c n}^{\sqrt{\alpha^{*}}=8,063 \sqrt{0,303}=4,436}$. La correspondiente media es $\mu_{Y}=69,719$. Los resultados se ilustran en la siguiente gráfica:

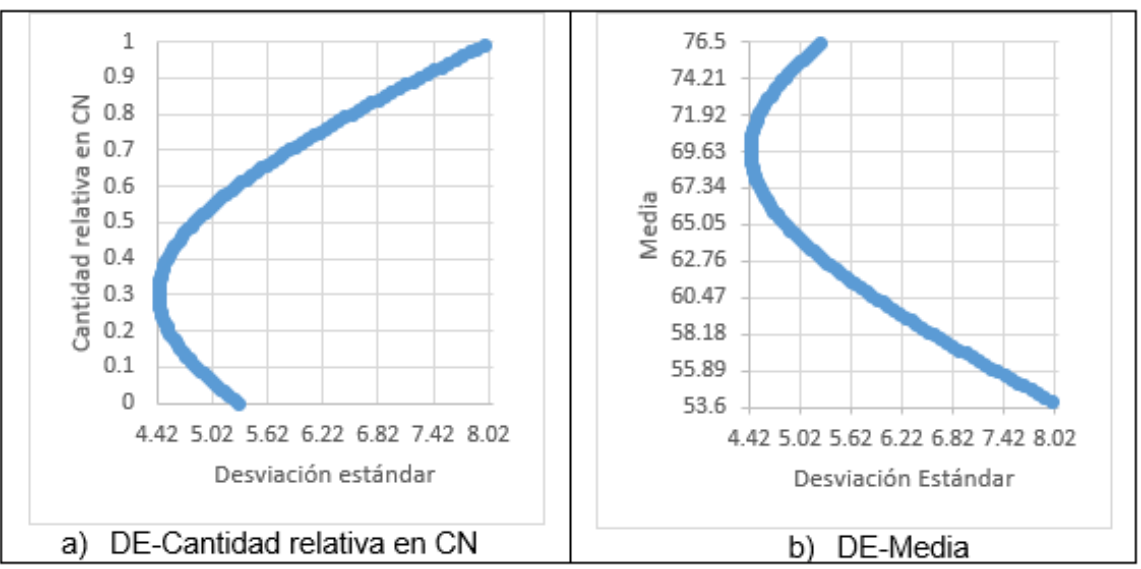

Gráfica 10. CTNGE-portafolio: Central nuclear y ciclo combinado Fuente: Elaboración propia con datos obtenidos de la simulación.

Dada la relación que existe entre la cantidad relativa asignada a la central nuclear, $\alpha$, y la "frontera eficiente" se tiene lo siguiente: las gráficas 16a y 16b indican que la frontera eficiente queda definida por el intervalo $0,303 \leq \alpha \leq 1$, donde la DE del CTNGE-portafolio se encuentra en el intervalo $4,436 \leq \sigma_{Y}^{*} \leq 8,063$, mientras que la correspondiente media se encuentra en el intervalo $69,719 \geq \mu_{Y} \geq 53,785$. No obstante, esta "frontera eficiente" da lugar a que el CTNGE-portafolio pueda tener una media muy baja, pero a un riesgo muy alto cuando $\alpha$ es cercano a 1 .

Se puede tener una "frontera eficiente" que considere un riesgo considerablemente menor aplicando el segundo resultado de la Proposición 3. Se calcula $\alpha_{L}=2 \alpha^{*} 2(0,303)=$ 0,606. De este modo, el riesgo del CTNGE-portafolio será menor o igual al de la tecnología menos riesgosa, ciclo combinado, $\sigma_{Y} \leq \sigma_{c c}$, cuando $0 \leq \alpha \leq 0,606$. Por consiguiente, el análisis sugiere que la familia de portafolios factibles, que tienen el CTNGE con menor media y menor DE, se tiene en la intersección de los dos intervalos anteriores, cuando la central nuclear recibe una cantidad relativa en el intervalo factible $0,303 \leq \alpha \leq 0,606$. Para dichos valores de $\alpha$, la DE del CTNGE-portafolio se encuentra en el intervalo 4,436 $\leq$ $\sigma_{Y}^{*} \leq 5,313$, mientras que la correspondiente media está en el intervalo $69,719 \geq \mu_{Y} \geq$ 62,878 . 


\subsection{Análisis de los Resultados}

Los resultados del análisis de portafolio se presentan en la tabla 17. A continuación se emplean para encontrar la mezcla de tecnologías de carga base que tenga el CTNGE con menor media y menor DE. Hay que recordar que el valor de $\alpha$ se refiere a la cantidad relativa asignada a la tecnología más riesgosa, indicada con $\left(^{*}\right)$, de cada portafolio.

Tabla 7. DE y medias de los CTNGE-portafolio

\begin{tabular}{|c|c|c|c|}
\hline & TC*-CC & TC $^{*}$-CN & CN $^{*}$-CC \\
\hline Intervalo factible & $0 \leq \alpha \leq 0,207$ & $0 \leq \alpha \leq 0,376$ & $0,303 \leq \alpha \leq 0,606$ \\
\hline Media & $76,559 \leq \mu_{Y} \leq 79,308$ & $53,785 \leq \mu_{Y} \leq 67,661$ & $69,719 \geq \mu_{Y} \geq 62,878$ \\
\hline Desv. Est. & $5,313 \geq \sigma_{Y}^{*} \geq 4,73$ & $8,063 \geq \sigma_{Y}^{*} \geq 6,369$ & $4,436 \leq{ }^{*} \leq 5,313$ \\
\hline
\end{tabular}

Fuente: Elaboración propia con datos obtenidos de la simulación.

Para comparar los portafolios, se adapta el criterio descrito por Jansen et al. (2006), que establece que, desde un punto de vista social, se busca el portafolio que brinde el CTNGE con menor media a un aceptable nivel de riesgo.

Partiendo de lo anterior, primero se comparan los portafolios que incluyen a la termoeléctrica de carbón. El CTNGE del portafolio que incluye a la termoeléctrica de carbón y a la central nuclear es más riesgoso que el portafolio que incluye a la termoeléctrica de carbón y al ciclo combinado. No obstante, este último portafolio tiene una media considerablemente mayor. La diferencia entre las medias de los CTNGE de estos portafolios se encuentra entre $\$ 8.898$ y $\$ 25.525$ dólares/MWh, que es muy grande, dada la producción de MWh de cada central. Por tal, dada la diferencia entre las medias de los CTNGE, se puede optar por el portafolio cuyo CTNGE tenga menor media, pero con mayor riesgo: Termoeléctrica de carbón y central nuclear.

Segundo, se compara el portafolio que incluye a la central nuclear y al ciclo combinado con los dos portafolios restantes. La media del CTNGE del portafolio que incluye a la central nuclear y al ciclo combinado es considerablemente menor a la media del CTNGE del portafolio que incluye a la termoeléctrica de carbón y al ciclo combinado. Por otro lado, dependiendo del valor de $\alpha$, la media del CTNGE del portafolio que incluye a la central nuclear y al ciclo combinado puede ser mayor que la media del CTNGE del portafolio que incluye la termoeléctrica de carbón y a la central nuclear. Siendo este último portafolio el más riesgoso de todos.

El análisis media-varianza sugiere que el portafolio que incluye a la central nuclear y al ciclo combinado es el que tiene el CTNGE con menor media y menor varianza. De hecho, para valores adecuados de la cantidad relativa asignada a la central nuclear, $\alpha$, se puede lograr que este portafolio sea menos riesgoso que el que incluye a la termoeléctrica de carbón y al ciclo combinado. Esto es, si la cantidad relativa asignada a la central nuclear, $\alpha$, seencuentraenelintervalo, $0.303 \leq \alpha \leq 0,473$, la DE del CTNGE-portafolio estará en el intervalo $4,436 \leq \sigma_{Y}^{*} \leq 4,73$, mientras que la media correspondiente se encontrará en el intervalo $69,719 \geq \mu_{Y} \geq 65,814$. Si bien se aumenta el límite inferior de la media del CTNGE de este portafolio, se mantiene competitivo con la media del CTNGE del portafolio que incluye a la termoeléctrica de carbón y a la central nuclear.

Es importante destacar dos puntos. Primero, en los portafolios que incluyen a la termoeléctrica de carbón, a esta tecnología se le asigna una baja cantidad relativa. A lo más, un $20.7 \%$ cuando el portafolio incluye al ciclo combinado y un máximo del $37.6 \%$ cuando el portafolio incluye a la central nuclear. Esto es recomendable en términos sociales ya que, al ser la termoeléctrica de carbón la tecnología que más contamina, el asignarle un porcentaje bajo de participación, tendrá como consecuencia, una menor afectación social. Segundo, en los portafolios que incluyen a la central nuclear, a esta tecnología se le asigna una alta cantidad relativa. A lo más, un $62.4 \%$ cuando el portafolio incluye a la termoeléctrica de carbón, y entre el $30.3 \%$ y el $60.6 \%$ ( $47.3 \%$ cuando se quiere reducir aún más el riesgo) cuando el portafolio incluye al ciclo combinado. 
El análisis sugiere que, al generar electricidad, conviene dejar fuera a la termoeléctrica de carbón y centrarse en dos tecnologías: ciclo combinado y central nuclear, asignando a ésta última una proporción mayor de capital. Con lo que se garantizan menores emisiones de $\mathrm{CO}_{2}$.

\section{Conclusiones}

El presente análisis sugiere que el CTNGE se puede modificar significativamente al incluir el costo del $\mathrm{CO}_{2}$. De acuerdo con los resultados obtenidos, se puede concluir que:

1. El CTNGE de la termoeléctrica de carbón, además de tener la mayor media, es el más riesgosa al presentar la mayor desviación estándar. La media del CTNGE de la central nuclear es menor a la media del CTNGE del ciclo combinado. Sin embargo, la central nuclear muestra un CTNGE más riesgoso que el correspondiente CTNGE del ciclo combinado.

2. La distribución acumulada (DA) del CTNGE de la termoeléctrica de carbón muestra dominancia estocástica de primer orden sobre la DA del CTNGE de ciclo combinado y la central nuclear. Es decir, la termoeléctrica de carbón produce, sin ambigüedades, los mayores CTNGE. En segundo lugar, se encuentran los CTNGE del ciclo combinado. Siendo la central nuclear la tecnología que produce los menores CTNGE.

3. El CTNGE de la termoeléctrica de carbón es muy sensible ante cambios en el precio de las emisiones de $\mathrm{CO}_{2}$ y del costo unitario de inversión.

4. El CTNGE de la central de ciclo combinado es más sensible a cambios en el precio de las emisiones de $\mathrm{CO}_{2}$.

5. El parámetro que mayor impacto tiene sobre el CTNGE de la central nuclear es el costo unitario de inversión.

6. El CTNGE-portafolio, así como su media y su DE quedan definidos por la cantidad relativa asignada a la tecnología más riesgosa.

7. El portafolio que incluye a la central nuclear y al ciclo combinado es el que tiene el CTNGE con menor media y menor varianza.

8. Al generar electricidad, conviene dejar fuera a la termoeléctrica de carbón y centrarse en dos tecnologías: ciclo combinado y central nuclear, asignando a ésta última una proporción mayor de capital.

\section{Recomendaciones y Trabajos futuros}

Partiendo de las conclusiones, se recomienda que, para garantizar menores emisiones de $\mathrm{CO}_{2}$, la generación de electricidad debe estar centrada en dos tecnologías de carga base: ciclo combinado y central nuclear, asignando a ésta última una proporción mayor de capital.

Esta recomendación es muy relevante para el contexto mexicano, debido a que en 2017 solo el $3 \%$ de la energía eléctrica fue generada en una central nuclear, mientras el $50 \%$ fue generada por centrales de ciclo combinado. Adicionalmente, la participación de las centrales de ciclo combinado será mayor en la generación de electricidad (Secretaría de Energía, 2018). Lo que tendrá como consecuencia directa una mayor afectación social a través de las emisiones de $\mathrm{CO}_{2}$ al ambiente.

En el presente trabajo se analizaron tecnologías de carga base de generación de electricidad. Una extensión directa será: aplicar la metodología del CTNG a tecnologías de generación de electricidad de tipo intermitente como la fotovoltaica y eólica. Emplear 
simulación Monte Carlo para generar y comparar los CTNG de las diferentes tecnologías de carga base y las intermitentes.

En el análisis del presente trabajo se asumió una covarianza de cero entre los CTNGE de las diferentes tecnologías. Una extensión directa será simular la correlación que existe entre los CTNGE de las diferentes tecnologías.

\section{Referencias}

Annual Energy Outlook. (2017) [AEO (2017)]. Editado por la U.S. Energy Information Administration. Recuperado el 15 de junio de 2017, de https://www.eia.gov/outlooks/archive/aeo17/pdf/ 0383(2017) .pdf

Awerbuch, S. y Berger, M. (2003). Applying portfolio Theory to EU Electricity Planning and PolicyMaking. IEA/EET Working Paper. IEA. Paris.

CENACE, Centro Nacional de Control de Energía. Secretaría de Energía. Recuperado el 15 de octubre de 2017, de https://www.gob.mx/cenace

Comisión Federal de Electricidad. (2014). Costos y Parámetros de Referencia para la Formulación de Proyectos de Inversión del Sector Eléctrico (COPAR). Subdirección de Programación, Comisión Federal de Electricidad.

Dixit, A. y Pindyck, R. S. (1994). Investment under uncertainty. Princeton University.

Eschenbach, T. G. (2006). Technical note: Constructing tornado diagrams with spreadsheets. The Engineering Economists 51:195-204.

Forbes C., Evans, M., Hastings, N. y Peacock, B. (2011). Statistical Distributions, 4th ed. New York: Wiley.

Freund, J. E., Miller, I. y Miller, M. (2000). Estadística matemática con aplicaciones. Pearson educación.

Gómez-Ríos, M. d. (2016). Aplicación de modelos estocásticos en centrales nucleares generadoras de energía eléctrica para detectar el impacto que tiene la volatilidad de los mercados financieros en los costos nivelados de generación. En C. IMEF, Tópicos actuales de Finanzas. pp 220 - 260.

Gómez-Ríos, M.-d.-C. (2008). La Energía Nuclear: una alternativa de generación de energía eléctrica de carga base en México. Tesis de Doctorado, México, Universidad Anáhuac México, campus Norte.

Hrafnkelsson, B., Oddsson, V., y Unnthorsson, R. (2016). .A Method for Estimating Annual Energy Production Using Monte Carlo Wind Speed Simulation." Energies 9, no. 4: 286.

ICF Consulting Canada Inc. (2017). Long-Term Carbon Price Forecast Report. May 31, 2017. Recuperado el 15 de marzo de 2018, de https://www.oeb.ca/sites/default/files/uploads/OEB-LTCPF-Report20170531.pdf

International Energy Agency. (2017). $\mathrm{CO}_{2}$ emissions from fuel combustion: overview (2017 edition).

International Energy Agency. (2014). International Energy Agency 2013 Annual Report.

International Energy Agency. (2005). Act locally, trade globally. Emissions trading for climate policy.

International Energy Agency. (2001). International Emission Trading from Concept to Reality.

Jansen, J.C., Beurskens, L.W.M., y Tilburg, X.V. (2006). Application of portfolio analysis to the Dutch generating mix. ECN report C-05-100. Energy research council of Netherlands.

Karkhov, A. (2002). Economic evaluation of bids for nuclear power plants. Atomnaya Tekhnika za Rubezhom, $23-26$.

Khindanova, I. (2013). A Monte Carlo Model of a Wind Power Generation Investment. The Journal of Applied Business and Economics, 15(1), 94.

Kienzle F. y Andersson, G. (2008). "Efficient multi-energy generation portfolios for the future," 4th Annu. Carnegie Mellon Conf. Elect. Ind. pp. 1-18.

Mas-Colell, A., Whinston, M. D. y Green, J. R. (1995). Microeconomic theory. Oxford University Press.

Markowitz, H. M. (1952), "Portfolio Selection", Journal of Finance, Vol. 7, pp 77-91.

Nuclear Energy Agency (NEA) e International Energy Agency (IEA). (2015). Projected Costs of Generating Electricity.

Nuclear Energy Agency. (2003). Nuclear Electricity Generation: What are the external costs? NEA 4372.

Organisation for Economic Co-operation and Development. (2017). The arrangement for officially supported export credits.

Secretaría de Hacienda y Crédito Público, (2017). Informe Semanal del Vocero.

Ramirez, J. R., Alonso, G., Perry, R. y Ortiz, J. (2006). Assessment of MOX fuel assembly design for a BWR mixed reload. Nuclear Technology. Vol. 156.

Rode, D., Fishbeck, P. y Dean, S. (2001), "Monte Carlo Methods for Appraisal and valuation: A Case Study of a Nuclear Power Plant", Journal of Structured and Project Finance, 7:3. p. 38-48.

Roques, F. A., Newbery, D. M. y Nuttall, W. J. (2008), "Fuel mix diversification incentives in liberalized electricity markets: A Mean-Variance Portfolio theory approach", Energy Economics, Volume 30, Issue 4: 1831-1849. 
Roques, F. (2006). Power generation investments in liberalised markets: methodologies to capture risk, flexibility, and portfolio diversity. Économies et Sociétés, 40(10/11), 1563.

Roques, F. A., Nuttall, W. J., Newbery, D. M., de Neufville, R. y Connors, S. (2006) "Nuclear Power: a Hedge against Uncertain Gas and Carbon Prices?" The Energy Journal, 27 (4): 1-24

Ross, S. (1999). Simulación. Prentice Hall.

Secretaría de Energía (2018). Programa del Desarrollo del Sistema Eléctrico Nacional 2018-2032.

Spadaro J. V., Langlois, L. y Hamilton, B. (2000) "Assessing the difference". IAEA Bull. 2000;42 (2):19-24

Vithayasrichareon, P., MacGill, I.F. y Wen, F.S. (2010a). Electricity generation portfolio evaluation for highly uncertain and carbon constrained future electricity industries. IEEE Power and Energy Society General Meeting.

Vithayasrichareon, P., MacGill, I.F. y Wen, F. (2010b). Electricity Generation Portfolio Analysis for Coal, Gas and Nuclear Plant under Future Uncertainties. 4th IASTED Asian Conference on Power and Energy Systems.

\section{Apéndice}

Demostración de la Proposición 3: Suponemos que el CTNGE de la tecnología 1 es más riesgoso que el CTNGE de la tecnología 2, $\sigma_{1}^{\geq} \sigma_{2}$ (o, alternativamente, $\sigma_{1}^{2} \geq \sigma_{2}^{2}$.). Para $\sigma_{1,2}=0$ y $0 \leq \alpha \leq 1$. Demostración de a) para analizar el riesgo del CTNGEportafolio se analiza primero el comportamiento de su varianza, $\sigma_{Y}^{2}$, de acuerdo con el capital relativo asignado a la tecnología más riesgosa, la 1, dada por $\alpha$. Para encontrar el punto óptimo, buscamos primero los puntos críticos. (c. p. o.)

$$
\begin{gathered}
\frac{d \sigma_{Y}^{2}}{d \alpha}=2 \alpha_{1}^{\sigma 2}-2(1-\alpha)_{2}^{\sigma} 2=0 \\
\Rightarrow \alpha_{1}^{\sigma 2}-(1-\alpha)_{2}^{\sigma 2}=0 \\
\Rightarrow \alpha_{1}^{\sigma 2}-\sigma_{2}^{2}+\alpha \sigma_{2}^{2}=0 \\
\Rightarrow \alpha^{\left(\sigma_{1}^{2}+\sigma_{2}^{2}\right)=\sigma_{2}^{2}} \\
\Rightarrow \alpha^{*}=\frac{\sigma_{2}^{2}}{\sigma_{1}^{2}+\sigma_{2}^{2}} .
\end{gathered}
$$

como $\sigma_{1} \geq \sigma_{2}$, entonces $\alpha^{*} \leq \frac{1}{2}$. Hay que verificar que el punto crítico en efecto es un mínimo [c. s. o.]

$$
\frac{d^{2} \sigma_{Y}^{2}}{d \alpha^{2}}=2 \sigma_{1}^{2}+2 \sigma_{2}^{2}>0
$$

Por lo que $\sigma_{Y}^{2}$ tiene un mínimo en $\alpha^{*}=\frac{\sigma_{2}^{2}}{\sigma_{1}^{2}+\sigma_{2}^{2}}$. Entonces, el valor mínimo de la varianza del CTNGE diversificado, $\sigma_{Y}^{2 *}$, es

$$
\begin{gathered}
\sigma_{Y}^{2 *}=\left[\frac{\sigma_{2}^{2}}{\sigma_{1}^{2}+\sigma_{2}^{2}}\right]^{2} \sigma_{1}^{2}+\left(1-\frac{\sigma_{2}^{2}}{\sigma_{1}^{2}+\sigma_{2}^{2}}\right)^{2} \sigma_{2}^{2}, \\
\sigma_{Y}^{2 *}=\frac{\sigma_{2}^{4}}{\left[\sigma_{1}^{2}+\sigma_{2}^{2}\right]^{2}} \sigma_{1}^{2}+\frac{\sigma_{1}^{4}}{\left[\sigma_{1}^{2}+\sigma_{2}^{2}\right]^{2}} \sigma_{2}^{2}, \\
\sigma_{Y}^{2 *}=\frac{\sigma_{1}^{2} \sigma_{2}^{2}\left[\sigma_{1}^{2}+\sigma_{2}^{2}\right]}{\left[\sigma_{1}^{2}+\sigma_{2}^{2}\right]^{2}}, \\
\sigma_{Y}^{2 *}=\frac{\sigma_{1}^{2} \sigma_{2}^{2}}{\sigma_{1}^{2}+\sigma_{2}^{2}}, \\
\sigma_{Y}^{2 *}=\sigma_{1}^{2} \alpha^{*} .
\end{gathered}
$$

como $\alpha^{*} \leq \frac{1}{2}$, entonces, $\sigma_{Y}^{2 *} \leq \frac{\sigma_{1}^{2}}{2}$. Por consiguiente, la desviación estándar, $\sigma_{Y}$, alcanza su mínimo, $\sigma_{Y}^{*}=\sigma_{1}^{\sqrt{\alpha^{*}} \leq \frac{\sigma_{1}}{\sqrt{2}}}$, cuando $\alpha^{*}=\frac{\sigma_{2}^{2}}{\sigma_{1}^{2}+\sigma_{2}^{2}}$.

de b) Aquí demostramos que es posible encontrar la distribución exacta de capital, $\alpha_{L}$, que permita que el CTNGE diversificado sea menor o igual de riesgoso que la tecnología menos riesgosa, $\sigma_{Y} \leq \sigma_{2}$, Comenzamos por encontrar $\alpha_{L}$ tal que $\sigma_{Y}=\sigma_{2}$. Así

$$
\begin{gathered}
\sigma_{\bar{Y}} \overline{\sqrt{\alpha^{2} \sigma_{1}^{2}+(1-\alpha)^{2} \sigma_{2}^{2}}}=\sigma_{2}, \\
\Rightarrow \alpha^{2} \sigma_{1}^{2}+(1-\alpha)^{2} \sigma_{2}^{2}=\sigma_{2}^{2}
\end{gathered}
$$




$$
\begin{gathered}
\Rightarrow \alpha^{2} \sigma_{1}^{2}+\left(\alpha^{2}-2 \alpha\right) \sigma_{2}^{2}=0, \\
\Rightarrow \alpha^{2} \sigma_{1}^{2}+\alpha(\alpha-2) \sigma_{2}^{2}=0, \\
\Rightarrow \alpha_{L}\left[\alpha_{L} \sigma_{1}^{2}+\left(\alpha_{L}-2\right) \sigma_{2}^{2}\right]=0, \\
\Rightarrow \alpha_{L}=0 \mathrm{y} / \hat{\mathrm{o}}\left[\alpha_{L} \sigma_{1}^{2}+\left(\alpha_{L}-2\right) \sigma_{2}^{2}\right]=0, \\
\Rightarrow \alpha_{L}\left[\sigma_{1}^{2}+\sigma_{2}^{2}\right]=2 \sigma_{2}^{2}, \\
\Rightarrow \alpha_{L}=\frac{2 \sigma_{2}^{2}}{\sigma_{1}^{2}+\sigma_{2}^{2}}=2 \alpha^{*} .
\end{gathered}
$$

Por lo que, se cumple que $\sigma_{2} \geq \sigma_{Y} \geq \sigma_{Y}^{*}$ para $0 \leq \alpha \leq 2 \alpha^{*}$. Es decir, en este intervalo, el riesgo del CTNGE diversificado es menor o igual al riesgo de la tecnología menos riesgosa. Por tal, si $\alpha=0$ ó $\alpha=\frac{2 \sigma_{2}^{2}}{\sigma_{1}^{2}+\sigma_{2}^{2}}$ se tiene que $\sigma_{Y}=\sigma_{2}$. Si $\alpha=1$, se tiene que $\sigma_{Y}=\sigma_{1}$. Lo que completa la demostración. 